\title{
Pathogenic Determinants of the Mycobacterium kansasii Complex: An Unsuspected Role for Distributive Conjugal Transfer
}

\author{
Florian Tagini ${ }^{1}$, Trestan Pillonel ${ }^{1}$ (D), Claire Bertelli ${ }^{1}$, Katia Jaton ${ }^{1}$ and Gilbert Greub ${ }^{1,2, *(D)}$ \\ 1 Department of Laboratory Medicine, Institute of Microbiology, Lausanne University Hospital and University \\ of Lausanne, 1011 Lausanne, Switzerland; florian.tagini@chuv.ch (F.T.); trestan.pillonel@chuv.ch (T.P.); \\ claire.bertelli@chuv.ch (C.B.); katia.jaton-ogay@chuv.ch (K.J.) \\ 2 Division of Infectious Diseases, Department of Medicine, Lausanne University Hospital, \\ 1011 Lausanne, Switzerland \\ * Correspondence: gilbert.greub@chuv.ch
}

check for updates

Citation: Tagini, F.; Pillonel, T.; Bertelli, C.; Jaton, K.; Greub, G. Pathogenic Determinants of the Mycobacterium kansasii Complex: An Unsuspected Role for Distributive Conjugal Transfer. Microorganisms 2021, 9, 348. https://doi.org/ $10.3390 /$ microorganisms 9020348

Academic Editor: Federico Giannoni Received: 14 January 2021

Accepted: 5 February 2021

Published: 10 February 2021

Publisher's Note: MDPI stays neutral with regard to jurisdictional claims in published maps and institutional affiliations.

Copyright: (c) 2021 by the authors. Licensee MDPI, Basel, Switzerland. This article is an open access article distributed under the terms and conditions of the Creative Commons Attribution (CC BY) license (https:// creativecommons.org/licenses/by/ $4.0 /)$.

\begin{abstract}
The Mycobacterium kansasii species comprises six subtypes that were recently classified into six closely related species; Mycobacterium kansasii (formerly M. kansasii subtype 1), Mycobacterium persicum (subtype 2), Mycobacterium pseudokansasii (subtype 3), Mycobacterium ostraviense (subtype 4), Mycobacterium innocens (subtype 5) and Mycobacterium attenuatum (subtype 6). Together with Mycobacterium gastri, they form the M. kansasii complex. M. kansasii is the most frequent and most pathogenic species of the complex. M. persicum is classically associated with diseases in immunosuppressed patients, and the other species are mostly colonizers, and are only very rarely reported in ill patients. Comparative genomics was used to assess the genetic determinants leading to the pathogenicity of members of the M. kansasii complex. The genomes of 51 isolates collected from patients with and without disease were sequenced and compared with 24 publicly available genomes. The pathogenicity of each isolate was determined based on the clinical records or public metadata. A comparative genomic analysis showed that all M. persicum, M. ostraviense, $M$ innocens and M. gastri isolates lacked the ESX-1-associated EspACD locus that is thought to play a crucial role in the pathogenicity of $M$. tuberculosis and other non-tuberculous mycobacteria. Furthermore, M. kansasii was the only species exhibiting a 25-Kb-large genomic island encoding for 17 type-VII secretion system-associated proteins. Finally, a genome-wide association analysis revealed that two consecutive genes encoding a hemerythrin-like protein and a nitroreductase-like protein were significantly associated with pathogenicity. These two genes may be involved in the resistance to reactive oxygen and nitrogen species, a required mechanism for the intracellular survival of bacteria. Three non-pathogenic $M$. kansasii lacked these genes likely due to two distinct distributive conjugal transfers (DCTs) between M. attenuatum and M. kansasii, and one DCT between M. persicum and M. kansasii. To our knowledge, this is the first study linking DCT to reduced pathogenicity.
\end{abstract}

Keywords: whole-genome sequencing; virulence; virulence factor; nontuberculous mycobacteria; genomics; conjugative plasmid; horizontal gene transfer; conjugation; transduction; mosaicism

\section{Introduction}

Mycobacterium kansasii, a member of non-tuberculous mycobacteria (NTM), is an environmental bacterium that can cause pulmonary diseases mostly in patients with immunosuppression or underlying lung diseases. Although it depends on the geographical area, M. kansasii is usually classed as the second or third most common NTM isolated from patients [1,2]. The diagnosis of pulmonary $M$. kansasii infections relies on clinical, radiological and microbiological findings [3]. Clinical findings include chronic cough, sputum production, fatigue, fever, hemoptysis, chest pain and weight loss. Chest X-ray and CT-Scan classically show cavitation, nodules or bronchiectasis. Finally, microbiological criteria for the diagnosis are (i) two positive cultures from two separated sputum samples, 
or (ii) positive culture from one bronchoalveolar lavage (BAL), or (iii) mycobacterial histopathological findings on the lung biopsies and one positive culture (of sputum or BAL). In addition, $M$. kansasii can also cause skin, soft tissues, and osteoarticular infections in both immunocompetent and immunocompromized patients [4]. In some very sporadic cases, M. kansasii can also be found in the urine [5], sometimes with pathogenic manifestations [6].

$M$. kansasii isolates was previously classified into seven different subtypes, based on PCR and restriction fragment length polymorphisms (RFLP) of the gene $h s p 65[7,8]$. Discrimination using other typing schemes, offering the advantage of using only one restriction enzyme, have also been proposed [9]. A recent whole-genome sequence analysis showed that each subtype, except subtype 7 (not available for the study), corresponds to new species-level lineages of the $M$. kansasii complex [10]. The M. kansasii species is now restricted to subtype 1 , which includes the most common and pathogenic strains for both immunocompetent and immunocompromised patients [11]. Conversely, subtype 2, renamed M. persicum, is found mainly in $\mathrm{HIV}^{+}$patients $[10,12]$. The four other subtypes-subtype 3, 4, 5 and 6, reclassified as $M$. pseudokansasii, M. ostraviense, $M$. innocens and $M$. attenuatum, respectively - are rarely associated with diseases and mostly remain colonizers [10,13].

M. kansasii shares many virulence factors with $M$. tuberculosis. For instance, $M$. kansasii encodes the type-VII secretion system ESX-1 locus, involved in secretion of several effectors like EsxA (ESAT-6) and EsxB (CFP-10), through its ESX conserved components (called Ecc proteins) [14]. ESX-1-secreted proteins are thought to modulate the activation of macrophages, allow phagosomal escape and induce necrosis/apoptosis [15]. EsxA is a helix-turn-helix hairpin $6 \mathrm{kDa}$ protein, whose length matchds the thickness of eukaryotic cells' membranes, and might form insertions that could disrupt the phagosomal membranes [16]. In addition, another operon encoding the ESX-1-secreted effectors EspA, EspC and EspD was reported in M. kansasii [14] and is absent from non-pathogenic slow-growing mycobacteria [15]. The espACD operon was thus hypothesized to be a mycobacterial pathogenicity island $[17,18]$. Secretion of EspA and EspC is co-dependent on the reciprocal secretion of EsxA and EsxB, supporting the role of the espACD operon in ESX-1 function [19]. In vivo experiments and Phagosomal Perturbation Assay in THP-1 macrophages showed that $M$. kansasii can disrupt the phagosomal membrane and exhibited similar bacterial loads as M. tuberculosis [14]. Phagosomal disruption and translocation to the cytosol, a crucial virulence mechanism, was shown to occur for a $M$. kansasii strain and not for an environmental $M$. innocens strain [20]. M. kansasii escape was further confirmed by phagosomal perturbation FRET-based assays [14]. In addition, $M$. kansasii strains produced higher amounts of Esx-B (CFP-10) than M. pseudokansasii and M. ostraviense strains [21]. However, these results were obtained by Western blot from culture medium and could have been influenced by (i) the growth phase that could affect the production of Esx-B and (ii) the use of monoclonal antibodies despite protein sequences are different between subtypes. Finally, pathogenic $M$. kansasii and M. pseudokansasii strains were able to grow better than non-pathogenic M. pseudokansasii and M. attenuatum in Acanthamoeba castellanii, suggesting an increased resistance to phagocytic cells [22].

Transformation, transduction and conjugation are the three primary mechanisms of horizontal gene transfer (HGT) in bacteria [23]. There is currently no evidence for recent or ongoing HGTs in members of the M. tuberculosis complex, except M. canettii [24,25]. However, HGTs occur widely in non-tuberculous mycobacteria. Natural transformation, characterized by the horizontal acquisition of usually small DNA fragments $(3-5 \mathrm{~Kb})$ by an active bacterial uptake, was reported in M. smegmatis and M. avium [26-28]. Transduction, mediated by bacteriophages, also occurs in the Mycobacterium genus [29,30]. Unlike conjugation, these two mechanisms do not require cell-to-cell contacts to transfer DNA. Conjugative plasmids have been described in M. marinum, M. kansasii, M. avium and M. yongonense, and encode both type-IV and type-VII secretion systems loci [31]. A plasmid conjugation event between M. avium and M. kansasii within a patient with disseminated mixed infection was recently reported and confirmed experimentally [32]. These plas- 
mids can carry a very diverse set of genes, but there is currently no evidence for a role in the pathogenicity of NTM $[14,31]$. To our knowledge, the only plasmid associated with virulence in mycobacteria is the non-conjugative plasmid found in $M$. ulcerans, the causative agent of Buruli ulcers [33]. Chromosomal transfers were reported decades ago in M. smegmatis [34], but were only recently better characterized as a powerful conjugative mechanism called distributive conjugal transfer (DCT) [35]. DCT occurs frequently among mycobacteria, transferring DNA from a donor cell to a recipient cell through a process involving the type-VII secretion system ESX-1 and ESX-4 [28]. Genomic DNA fragments of the donor cell ranging from 59 base pairs (bp) to more than $200 \mathrm{Kbp}$ can be integrated into the recipient cell by homologous recombination and generate both macro- and micromosaic genomes [28]. DCT drastically differs from the Hfr conjugation model, where a conjugative plasmid is integrated in the chromosome [36]. Hfr conjugal events can lead to transfers of genomic regions downstream of the integrated episome, but do no produce mosaic genomes as seen with DCT.

To test the hypothesis that pathogenicity in the M. kansasii complex is based on genetic determinants carved by evolutionary processes, we performed a comparative genome analysis of 75 strains with varying level of pathogenicity. Genetic variations, including single nucleotide variants and gene gain/loss, were associated with pathogenicity, and shaped by intra- and inter-species HGT events that occurred by distributive conjugal transfer.

\section{Materials and Methods}

\subsection{Strain Inclusion and Pathogenicity Classification}

This study included all strains belonging to the $M$. kansasii complex isolated from patients between 2007 and 2017 at the Lausanne University Hospital, a Swiss tertiary care hospital. Strains were classified into five different categories: 1, pathogenic; 2, probably pathogenic; 3, unknown; 4, probably non-pathogenic/colonizer; 5 , non-pathogenic/colonizer. The presence of a mycobacterial disease and its compliance with 2007's criteria of the American Thoracic Society (ATS) for pulmonary NTM diseases was assessed based on the available clinical data [3]. "Probably pathogenic" cases did not fulfil completely all the ATS criteria, but the pathogenicity was strongly suspected based on available clinical, microbiological and/or radiological data. "Probably non-pathogenic/colonizer" cases were cases for which clinical, microbiological and/or radiological data indicated that the strain was likely a colonizer, but for which detailed data were not available. In our cohort, osteoarticular manifestations of M. kansasii complex diseases were all considered pathogenic (category 1). Proportions of pathogenic strains in the different species were compared using the "N-1" chi-squared test [37].

This dataset was completed with strains from two different external sources. Five M. kansasii and six M. pseudokansasii strains available from Goy et al. [22] were included. The pathogenicity of those pulmonary isolates had already been determined according to the $1997^{\prime}$ s criteria of the ATS for mycobacterial diseases [11,38]. This study also included a single M. pseudokansasii strain (MK142) that was isolated from blood culture of a patient with disseminated mycobacterial infection and thus classified as pathogenic [22]. Moreover, all publicly available genomes identified as M. kansasii and M. gastri in the NCBI Taxonomy database were used for the comparative genome analysis (as available in June 2017). The pathogenicity of these isolates was determined based on available metadata and then determined as either "unknown", "probably pathogenic" or "probably non-pathogenic/colonizer". The genome of $M$. tuberculosis reference strain H37Rv (GCF_000195955.2) was added to the dataset to perform genomic comparisons and to root the phylogenetic tree of NTM strains.

\subsection{Bacterial Cultures, DNA Extraction and Sequencing}

Mycobacteria were grown in Mycobacterial Growth Indicator Tubes BD BACTEC MGIT $^{\mathrm{TM}}$, supplemented with MGIT ${ }^{\mathrm{TM}}$ OADC enrichment and MGIT ${ }^{\mathrm{TM}}$ PANTA $^{\mathrm{TM}}$ antibiotic mixture (Becton Dickinson, Franklin Lakes, NJ, USA). DNA was extracted using the Wizard 
Genomic DNA Purification System (Promega, Madison, WI, USA, ref. A1120), following the protocol for mycobacteria. Briefly, culture pellets were inactivated for $1 \mathrm{~h}$ at $95^{\circ} \mathrm{C}$. Pellets were then resuspended in a solution of $300 \mu \mathrm{L}$ of $50 \mathrm{mM}$ EDTA pH 8.0 and $50 \mu \mathrm{L}$ lysozyme $100 \mathrm{mg} / \mathrm{mL}$, and incubated for $1 \mathrm{~h}$ at $37^{\circ} \mathrm{C}$. After $2 \mathrm{~min}$ of centrifugation at $20,000 \times g$, supernatants were resuspended in solution of $500 \mu \mathrm{L}$ of Nuclei lysis, $100 \mu \mathrm{L}$ SDS $20 \%, 150 \mu \mathrm{L}$ EDTA $0.5 \mathrm{M} \mathrm{pH} \mathrm{8,} 20 \mu \mathrm{L}$ Proteinase $\mathrm{K} 20 \mathrm{mg} / \mathrm{mL}$ and $3 \mu \mathrm{L}$ RNAse A and incubated for (i) $1 \mathrm{~h}$ at $55^{\circ} \mathrm{C}$ and (ii) for $5 \mathrm{~min}$ at $70^{\circ} \mathrm{C}$. Approximately $150 \mu \mathrm{L}$ of microbeads $<106 \mu \mathrm{m}$ (Sigma, Saint-Louis, MO, USA, ref. G4649) were added before shaking the samples with FastPrep at $6800 \mathrm{rpm}$ for $3 \times 30 \mathrm{~s}$. Samples were centrifuged for $2 \mathrm{~min}$ at 20,000× $g$ before transferring the supernatant into a new tube. The protocol for bacteria was then continued (200 $\mu \mathrm{L}$ protein precipitation solution, precipitation step with isopropanol 100\% and washing steps with ethanol 70\%). For MiSeq sequencing (Illumina, San Diego, CA, USA), final elution was done in $30 \mu \mathrm{L}$ of $10 \mathrm{mM}$ TRIS/0.1 mM EDTA pH 8.0 and incubated for $1 \mathrm{~h}$ at $65{ }^{\circ} \mathrm{C}$. For Pacific Biosciences RS II sequencing (Menlo Park, CA, USA), elution was done in $30 \mu \mathrm{L}$ of $10 \mathrm{mM}$ TRIS $\mathrm{pH} 8.5$ and incubated overnight at $4{ }^{\circ} \mathrm{C}$.

Genomic libraries were prepared using Nextera XT library kit (Illumina, San Diego, CA, USA, ref. FC-131-1096) and size distributions were checked using an automated capillary electrophoresis instrument (Fragment Analyzer ${ }^{\mathrm{TM}}$ Automated CE System, Advanced Analytical, Agilent Technologies, Santa Clara, CA, USA). Whole genome sequencing was performed with a MiSeq sequencer (Illumina, San Diego, CA, USA) using either $150 \mathrm{bp}$ or 250 bp paired-end reads. Strain MK142 was sequenced with a Pacific Biosciences RS II sequencer using one SMRT cell version P6-C4 (Pacific Biosciences, Menlo Park, CA, USA) as described in Tagini et al. [10].

\subsection{Quality Controls, Assembly, Annotation and Comparative Genomic Analyses}

Read quality was assessed with FastQC v0.11.4 before and after trimming (Andrews S, available online at: http:/ / www.bioinformatics.babraham.ac.uk/projects/fastqc, accessed on 8 February 2021). Reads were trimmed with Trimmomatic v0.35 (MINLEN:60, LEADING:9, TRAILING:9, SLIDING-WINDOW:3:15) [39]. Assemblies were performed using SPAdes v3.9.0 [40] with k-mer sizes ranging from 41 to $127 \mathrm{bp}$. Contigs were reordered with Mauve software 13 February 2015 snapshot for Linux using M. kansasii ATCC 12478 as a reference [41]. Genomes were annotated using Prokka 1.12 [42].

For the comparative genomic analyses, a MySQL database was created as described in Tagini et al. to perform comparative analyses and browse gene annotation using a web interface [43]. Briefly, each coding sequence was BLASTed (using BLASTP algorithm) against the protein Cluster of Orthologous Genes (COG) database with e-value, amino acid identity and query coverage cut-offs of $10^{-5}, 20 \%$ and $50 \%$, respectively [44,45]. Kegg Orthology (KO) numbers were assigned using GhostKOALA v2.0 [46]. Protein domains were predicted using InterProScan v5.18-57.0 [47]. Orthologous and paralogous sequences were identified and clustered into so-called "orthogroups" using OrthoFinder v1.1.4 [48]. For each orthogroup, the amino acid sequences were aligned using Mafft v7.187 [49], and a maximum-likelihood phylogeny was built using RaxML v8.2.10 with parameters "-m PROTGAMMALG -p 12345" and "-f J" to compute the node supports [50]. Finally, available gene operons data were downloaded from the DOOR 2.0 database [51].

Multiple sequence alignments of single copy orthogroups conserved in all strains were concatenated into a single amino acid alignment that was used to reconstruct a maximum-likelihood phylogeny using FastTree v2.1.8 with parameters "-gamma -spr 4-mlacc 2-slownni" [52]. This phylogeny, the matrix of orthogroup presence/absence (including plasmid-encoded genes) as well as the degree of pathogenicity were used as input of a genome-wide association study (GWAS) on the whole dataset performed with TreeWAS v1.0 [53]. Furthermore, all genomes of the species $M$. kansasii were aligned using ParSNP v1.2 to identify conserved genomic regions (or core $M$. kansasii genome). Strains from other species were excluded to maximize the size of the core-genome alignment [54]. Then, trimmed reads of $M$. kansasii isolates were mapped onto identified conserved regions 
of strain ATCC 12478 using Snippy v3.1 [55]. A joint matrix of SNP presence/absence as well as single-copy orthogroups was built to test their association with the degree of pathogenicity and tissue tropism of M. kansasii strains only using TreeWAS v1.0 [53].

The presence of homologs to $M$. tuberculosis virulence factors in the orthogroups was assessed using the locus tags of (1) the genes listed in Forrellad et al. [56] and (2) the genes related to type-VII secretion systems loci (ESX-1, EspACD, ESX-2, ESX-3, ESX-4 and ESX-5) described in Simeone et al. [57]. Furthermore, genes specific to each species and each clinical presentation were identified and their annotation manually inspected to assess the presence of genes that could be involved in virulence.

\subsection{Detection of Distributive Conjugal Transfers between Members of the M. kansasii Complex}

Distributive conjugal transfers (DCTs) between strains of different species of the M. kansasii complex were identified by looking for genes exhibiting higher similarity between strains of different species than among strains of the same species. Practically, the mean amino acid identity with strains of the same species and with strains of the six other species of the M. kansasii complex was calculated for each locus. In the case of one-tomany orthology relationships, the amino acid identity of the closest orthologous gene was used for calculation. Then, if the mean amino acid identity with strains of another species was higher than with strains of the same species, the locus was considered as part of a candidate DCT region involving recombination between the considered strain and other species. Regions containing prophages were detected using the PHAge Search Tool Enhanced Release (PHASTER) [58,59]. All DCTs were represented using genoPlotR v0.8.7 [60].

\subsection{Detection of Conjugative Plasmids}

To detect conjugative plasmids in newly sequenced and publicly available draft genomes, complete plasmid sequences of M. kansasii pMK12478 and M. pseudokansasii pMK142 were used to perform BLASTN searches against the draft genomes (contig files) of the dataset with an e-value cutoff of 0.00001 . Contigs were extracted when returning more than one hit or if the alignment length exceeded $1000 \mathrm{bp}$. In addition, potential new conjugative plasmids were identified by searching for highly conserved genes of the mycobacterial conjugative machinery [31]. Contigs encoding homologs of the relaxase of pMK12478 (MKAN_RS28485) and VirB4 (MKAN_RS28085) were identified based on OrthoFinder grouping. In addition, open reading frames that could have been missed by the automated annotation were searched in contigs with TBLASTN. Since conjugative plasmids encode an additional type-VII secretion system locus, Hidden-Markov Model (HMM) searches for the ESX conserved components proteins and WxG100 effector proteins (like EsxA and EsxB) were performed using MacSyFinder v.1.0.4 with a custom database of published HMM models (TIGR03922, EccA; TIGR03919, EccB; TIGR03924, EccCa; TIGR03925, EccCb; TIGR03920, EccD; TIGR03923, EccE; TIGR03921, MycP; TIGR03930, WxG100 effector proteins) [61]. Variations in contig GC content and sequencing depth were investigated to identify contigs exhibiting similar characteristics as candidate plasmids sequences identified by homology. Only contigs encoding at least the extra type-VII secretion system plus a relaxase or a VirB4 homolog were considered in the comparative analysis. Episomes integrated in the middle of large contigs were not excluded. All plasmids were reannotated using Prokka v1.12 [42]. A MySQL plasmid database was created as described above with all detected conjugative plasmids as well as pRAW, a conjugative plasmid of M. marinum described by Ummels et al. that shares a common backbone structure with the plasmid of M. kansasii strain ATCC 12478 [31].

\section{Results}

\subsection{M. kansasii Is More Pathogenic than Other Members of the Complex}

Among the 40 strains isolated at the Lausanne University Hospital between 2007 and 2017, seventeen were isolated from patients presenting non-tuberculosis mycobacterial diseases (category 1 or 2) (Table 1). An immunosuppression was documented in seven 
of them. As expected, most patients with diseases had pulmonary clinical presentations. However, five bone and joint infections and one urinary tract infection were also reported, hence representing $35 \%$ of diseased patients. For nine patients, the strain pathogenicity could not be assessed due to the lack of clinical data. Finally, fourteen patients were only colonized with species of the M. kansasii complex (category 4 or 5), although two of them were immunosuppressed. MK8 and MK11, both M. pseudokansasii, were the only bacteria isolated from urine.

Table 1. Included strains of the M. kansasii complex and pathogenicity.

\begin{tabular}{|c|c|c|c|c|c|c|c|c|c|}
\hline Strain ID & Spec. & Age & Diagnosis & Co-Morbidities & P. & I. & O. & Species & Or. \\
\hline 1010001468 & Environm. & NA & NA & NA & 4 & NA & 0 & M. attenuatum & 0 \\
\hline MK136 & ND & ND & Colonization & ND & 5 & ND & 0 & M. attenuatum & 1 \\
\hline MK191 & ND & ND & Colonization & ND & 5 & ND & 0 & M. attenuatum & 1 \\
\hline MK41 & Bronch. asp. & 48 & Colonization & Lung transplanted & 5 & 1 & 0 & M. attenuatum & 2 \\
\hline NLA001001166 & ND & ND & Colonization & Bronchectasis & 4 & ND & 0 & M. attenuatum & 0 \\
\hline 1010001458 & Environm. & NA & NA & NA & 4 & NA & 0 & M. ostraviense & 0 \\
\hline DSM43505 & $\begin{array}{l}\text { Gastric } \\
\text { lavage }\end{array}$ & ND & Colonization & ND & 4 & ND & 0 & M. gastri & 0 \\
\hline 1010001454 & Environm. & NA & NA & NA & 4 & $\mathrm{NA}$ & 0 & M. innocens & 0 \\
\hline 1010001493 & Environm. & NA & NA & NA & 4 & $\mathrm{NA}$ & 0 & M. innocens & 0 \\
\hline MK13 & Sputum & 87 & ND & ND & 3 & ND & 0 & M. innocens & 2 \\
\hline 662 & $\begin{array}{l}\text { BAL/Bronch. } \\
\text { asp. }\end{array}$ & ND & Lung NTM disease & ND & 2 & ND & 0 & M. kansasii & 0 \\
\hline 824 & Sputum & ND & Lung NTM disease & ND & 2 & ND & 0 & M. kansasii & 0 \\
\hline 1010001495 & Environm. & NA & NA & NA & 4 & $\mathrm{NA}$ & 0 & M. kansasii & 0 \\
\hline $10 \mathrm{MK}$ & Sputum & ND & Lung NTM disease & ND & 2 & ND & 0 & M. kansasii & 0 \\
\hline $11 \mathrm{MK}$ & BAL & ND & Lung NTM disease & ND & 2 & ND & 0 & M. kansasii & 0 \\
\hline $1 \mathrm{MK}$ & Sputum & ND & Lung NTM disease & ND & 2 & ND & 0 & M. kansasii & 0 \\
\hline $4 \mathrm{MK}$ & Sputum & ND & Lung NTM disease & ND & 2 & ND & 0 & M. kansasii & 0 \\
\hline $5 \mathrm{MK}$ & BAL & ND & Lung NTM disease & ND & 2 & ND & 0 & M. kansasii & 0 \\
\hline $6 \mathrm{MK}$ & Sputum & ND & Lung NTM disease & ND & 2 & ND & 0 & M. kansasii & 0 \\
\hline 9MK & BAL & ND & Lung NTM disease & ND & 2 & ND & 0 & M. kansasii & 0 \\
\hline ATCC12478 & ND & ND & NTM disease & ND & 2 & ND & 0 & M. kansasii & 0 \\
\hline MK1 & BAL & 50 & Lung NTM disease & ND & 2 & ND & 0 & M. kansasii & 2 \\
\hline MK12 & Sputum & 19 & Lung NTM disease & - & 1 & 1 & 0 & M. kansasii & 2 \\
\hline MK135 & ND & ND & Lung NTM disease & ND & 1 & ND & 0 & M. kansasii & 1 \\
\hline MK156 & ND & ND & Lung NTM disease & ND & 1 & ND & 0 & M. kansasii & 1 \\
\hline MK17 & Bronch. asp. & 71 & Lung NTM disease & $\begin{array}{l}\text { Lung adenocarcinoma, } \\
\text { bronchectasis }\end{array}$ & 1 & 0 & 0 & M. kansasii & 2 \\
\hline MK18 & Bronch. asp. & 67 & ND & ND & 3 & ND & 0 & M. kansasii & 2 \\
\hline MK186 & ND & ND & Lung NTM disease & ND & 1 & ND & 0 & M. kansasii & 1 \\
\hline MK19 & Sputum & 68 & Lung NTM disease & ND & 2 & ND & 0 & M. kansasii & 2 \\
\hline MK190 & ND & $\mathrm{ND}$ & Lung NTM disease & ND & 1 & ND & 0 & M. kansasii & 1 \\
\hline MK20 & Sputum & 16 & Colonization & Cystic fibrosis & 5 & 0 & 0 & M. kansasii & 2 \\
\hline MK22 & Biopsy & 27 & $\begin{array}{l}\text { Tenosynovitis, } \\
\text { flexor of the 3rd } \\
\text { finger }\end{array}$ & $\begin{array}{l}\text { History of toxic } \\
\text { pneumonitis due to } \\
\text { hydrogen chroride } \\
\text { exposition }\end{array}$ & 1 & 0 & 1 & M. kansasii & 2 \\
\hline MK26 & Biopsy & 23 & $\begin{array}{l}\text { Finger infection (no } \\
\text { further } \\
\text { information) }\end{array}$ & ND & 1 & ND & 1 & M. kansasii & 2 \\
\hline
\end{tabular}


Table 1. Cont

\begin{tabular}{|c|c|c|c|c|c|c|c|c|c|}
\hline Strain ID & Spec. & Age & Diagnosis & Co-Morbidities & P. & I. & O. & Species & Or. \\
\hline MK28 & Bronch. asp. & 64 & ND & ND & 3 & ND & 0 & M. kansasii & 2 \\
\hline MK29 & BAL & 58 & ND & ND & 2 & ND & 0 & M. kansasii & 2 \\
\hline MK3 & BAL & 77 & ND & Lung adenocarcinoma & 4 & 0 & 0 & M. kansasii & 2 \\
\hline MK30 & Bronch. asp. & 67 & Colonization & $\begin{array}{l}\text { Lung adenocarcinoma, } \\
\text { COPD, liver cirrhosis, } \\
\text { valvular and rythmic } \\
\text { cardiopathy }\end{array}$ & 5 & 0 & 0 & M. kansasii & 2 \\
\hline MK31 & Bronch. asp. & 63 & ND & Rhumatoid arthritis & 3 & 1 & 0 & M. kansasii & 2 \\
\hline MK34 & Sputum & 82 & Lung NTM disease & $\begin{array}{l}\text { Rhumatoid arthritis with } \\
\text { lung involvement }\end{array}$ & 1 & 1 & 0 & M. kansasii & 2 \\
\hline MK36 & Sputum & 59 & Colonization & $\begin{array}{c}\text { Primary ciliary dyskinesia, } \\
\text { bronchectasis }\end{array}$ & 5 & 0 & 0 & M. kansasii & 2 \\
\hline MK38 & Surgical spec. & 47 & Sternitis & Rhumatoid arthritis & 1 & 1 & 1 & M. kansasii & 2 \\
\hline MK39 & Sputum & 49 & Lung NTM disease & ND & 2 & ND & 0 & M. kansasii & 2 \\
\hline MK40 & Surgical spec. & 49 & $\begin{array}{l}\text { Hand infection (no } \\
\text { further } \\
\text { information) }\end{array}$ & $\begin{array}{l}\text { Chronic hip prosthesis } \\
\text { infection with } \\
\text { Propionibacterium acnes }\end{array}$ & 1 & 0 & 1 & M. kansasii & 2 \\
\hline MK5 & Sputum & 32 & Lung NTM disease & ND & 2 & ND & 0 & M. kansasii & 2 \\
\hline MK52 & Sputum & 66 & Colonization & $\begin{array}{l}\text { Lung adenocarcinoma, } \\
\text { COPD }\end{array}$ & 5 & 0 & 0 & M. kansasii & 2 \\
\hline MK6 & Sputum & 73 & ND & ND & 3 & ND & 0 & M. kansasii & 2 \\
\hline MK7 & Sputum & 34 & Lung NTM disease & $\begin{array}{l}\text { Chronic hepatitis B treated } \\
\text { with alfa-2a peginterferon }\end{array}$ & 1 & 1 & 0 & M. kansasii & 2 \\
\hline MK83 & ND & ND & Lung NTM disease & ND & 1 & ND & 0 & M. kansasii & 1 \\
\hline SMC1 & ND & ND & Human-environ. & ND & 3 & ND & 0 & M. kansasii & 0 \\
\hline 1010001469 & Environm. & NA & NA & NA & 4 & NA & 0 & M. persicum & 0 \\
\hline $12 \mathrm{MK}$ & BAL & ND & Colonization & ND & 4 & ND & 0 & M. persicum & 0 \\
\hline $3 \mathrm{MK}$ & BAL & ND & Colonization & ND & 4 & ND & 0 & M. persicum & 0 \\
\hline $7 \mathrm{MK}$ & Sputum & ND & Colonization & ND & 4 & ND & 0 & M. persicum & 0 \\
\hline $8 \mathrm{MK}$ & BAL & ND & Colonization & ND & 4 & ND & 0 & M. persicum & 0 \\
\hline MK15 & $\begin{array}{l}\text { Endotracheal } \\
\text { secretions }\end{array}$ & 8 & Colonization & Still's disease & 5 & 1 & 0 & M. persicum & 2 \\
\hline MK16 & Sputum & 29 & Colonization & $\begin{array}{l}\text { Disseminated M. avium } \\
\text { infection }\end{array}$ & 3 & 1 & 0 & M. persicum & 2 \\
\hline MK4 & Bronch. asp. & 28 & NTM disease & $\begin{array}{l}\text { Acute lymphoblastic } \\
\text { leukemia, bone marrow } \\
\text { allografted }\end{array}$ & 1 & 1 & 0 & M. persicum & 2 \\
\hline MK42 & Surgical spec. & 32 & $\begin{array}{l}\text { Chronic olecranon } \\
\text { bursitis }\end{array}$ & ND & 1 & ND & 1 & M. persicum & 2 \\
\hline MK47 & Bronch. asp. & 77 & Lung NTM disease & Polymyalgia rhumatica & 1 & 1 & 0 & M. persicum & 2 \\
\hline MK53 & Sputum & 76 & ND & ND & 3 & ND & 0 & M. persicum & 2 \\
\hline MK54 & Bronch. asp. & 27 & Colonization & Bronchectasis & 5 & 0 & 0 & M. persicum & 2 \\
\hline MK11 & Urine & 27 & Colonization & Recurent UTI & 3 & 0 & 0 & $\begin{array}{l}\text { M. pseu- } \\
\text { dokansasii }\end{array}$ & 2 \\
\hline MK123 & ND & ND & Colonization & ND & 5 & ND & 0 & $\begin{array}{l}\text { M. pseu- } \\
\text { dokansasii }\end{array}$ & 1 \\
\hline MK142 & Blood culture & ND & $\begin{array}{l}\text { Bacteremia, } \\
\text { disseminated } \\
\text { disease }\end{array}$ & ND & 1 & ND & 0 & $\begin{array}{l}\text { M. pseu- } \\
\text { dokansasii }\end{array}$ & 1 \\
\hline MK151 & ND & ND & Colonization & ND & 5 & ND & 0 & $\begin{array}{l}\text { M. pseu- } \\
\text { dokansasii }\end{array}$ & 1 \\
\hline MK21 & Sputum & 52 & Colonization & $\begin{array}{c}\text { History of } \\
\text { community-acquired } \\
\text { pneumococcal pneumonia }\end{array}$ & 5 & 0 & 0 & $\begin{array}{c}\text { M. pseu- } \\
\text { dokansasii }\end{array}$ & 2 \\
\hline MK32 & Sputum & 80 & Colonization & $\begin{array}{l}\text { Small cell lung carcinoma, } \\
\text { history of radiation } \\
\text { pneumonitis and } M \text {. } \\
\text { tuberculosis infection }\end{array}$ & 5 & 0 & 0 & $\begin{array}{l}\text { M. pseu- } \\
\text { dokansasii }\end{array}$ & 2 \\
\hline
\end{tabular}


Table 1. Cont.

\begin{tabular}{|c|c|c|c|c|c|c|c|c|c|}
\hline Strain ID & Spec. & Age & Diagnosis & Co-Morbidities & P. & I. & O. & Species & Or. \\
\hline MK33 & Bronch. asp. & 60 & Colonization & Small cell lung carcinoma & 5 & 0 & 0 & $\begin{array}{l}\text { M. pseu- } \\
\text { dokansasii }\end{array}$ & 2 \\
\hline MK35 & Sputum & 47 & Colonization & $\begin{array}{l}\text { History of } M \text {. tuberculosis } \\
\text { infection, Barret's } \\
\text { oesophagus, rectal } \\
\text { adenocarcinoma }\end{array}$ & 5 & 0 & 0 & $\begin{array}{l}\text { M. pseu- } \\
\text { dokansasii }\end{array}$ & 2 \\
\hline MK43 & Bronch. asp. & 9 & Colonization & Miller-Dieker syndrome & 5 & 0 & 0 & $\begin{array}{l}\text { M. pseu- } \\
\text { dokansasii }\end{array}$ & 2 \\
\hline MK46 & Sputum & 30 & Colonization & $\begin{array}{l}\text { History of M. tuberculosis } \\
\text { infecction }\end{array}$ & 5 & 0 & 0 & $\begin{array}{l}\text { M. pseu- } \\
\text { dokansasii }\end{array}$ & 2 \\
\hline MK48 & Sputum & 33 & ND & ND & 3 & ND & 0 & $\begin{array}{l}\text { M. pseu- } \\
\text { dokansasii }\end{array}$ & 2 \\
\hline MK75 & ND & ND & Colonization & ND & 5 & ND & 0 & $\begin{array}{l}\text { M. pseu- } \\
\text { dokansasii }\end{array}$ & 1 \\
\hline MK8 & Urine & 51 & UTI & Renal transplanted & 1 & 1 & 0 & $\begin{array}{c}\text { M. pseu- } \\
\text { dokansasii }\end{array}$ & 2 \\
\hline
\end{tabular}

Strain ID, Strain identifier; Spec., specimen; ND, not determined; NA, not attributable; UTI, Urinary tract infection; BAL, Bronchoalveolar lavage; Bonch. asp., bronchial aspiration; Environm. Environmental; Diagnosis, diagnosis related to the isolated M. kansasii complex species; NTM, Non-tuberculosis mycobacteria; P., Pathogenicity; $1=$ pathogenic, $2=$ probably pathogenic, $3=$ unknown, $4=$ probably non-pathogenic/colonizer, $5=$ non-pathogenic/colonizer.; I. Immunosuppression, $1=$ yes, $0=$ no; O., Osteoarticular presentation, $1=$ yes, $0=$ no; Or., origin (source), 0 = publicly available genome from the NCBI, $1=$ strain used by Goy et al., $2=$ this study.

We compared the proportion of pathogenic/probably pathogenic strains between the most frequently isolated species, M. kansasii, M. persicum and M. pseudokansasii (Table 1). For the calculation, only strains recovered between 2007 and 2017 in the University hospital of Lausanne were used to avoid a selection bias (published genomes tend to be sequenced from more pathogenic strains). Strains with undetermined pathogenicity were excluded from the calculations. The proportions of pathogenic/probably pathogenic strains were $72 \%, 60 \%$ and $14 \%$ for $M$. kansasii (total = 18), M. persicum (total = 5) and M. pseudokansasii (total $=7$ ), respectively. The difference was only statistically significant between $M$. kansasii and M. pseudokansasii ( $p$-value of 0.01 ) likely because of the small sample size of M. persicum. These findings are congruent with a previous report [11].

It is interesting to note that MK48 and MK54 were recovered from two patients of the same family. However, transmission between patients or transmission from the same contaminated environment can be ruled out since MK48 strain clustered with M. pseudokansasii and MK54 with M. persicum strains. A familial genetic predisposition or predisposing disease (one of them was known for bronchiectasis) is thus the most likely hypothesis.

\subsection{Genomic Features and Core-Genome Phylogeny}

Genome sizes ranged from 6.03 to $6.9 \mathrm{Mb}$, which is $2 \mathrm{Mb}$ larger than M. tuberculosis (Figure S1) and the GC content was comprised between $65.8 \%$ and $66.2 \%$. The maximumlikelihood phylogeny, based on the concatenation of 2099 core orthogroup alignments (length of 729,256 amino acids), clustered each species of the $M$. kansasii complex into seven distinct clades (Figure 1B), consistent with a previous study [10]. M. kansasii and $M$. innocens isolates showed a greater genetic diversity than M. persicum, M. pseudokansasii or M. attenuatum. The heterogeneity of $M$. kansasii isolates may be due to the larger number of strains included as compared to other species and to particular local epidemiology, since three clades (MK30 to MK38, MK20 to MK18 and MK28 to MK29) contained only isolates from Lausanne. For $M$. innocens, the number of included strains is too small to be interpreted $(n=3)$. 
A

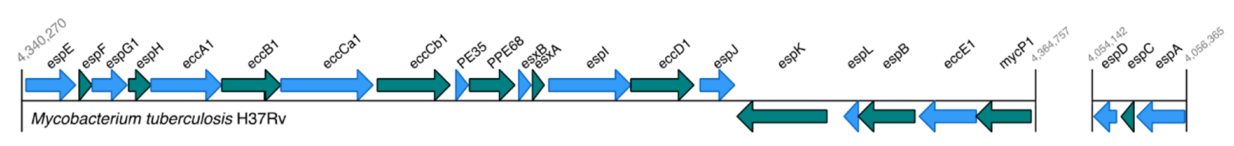

B

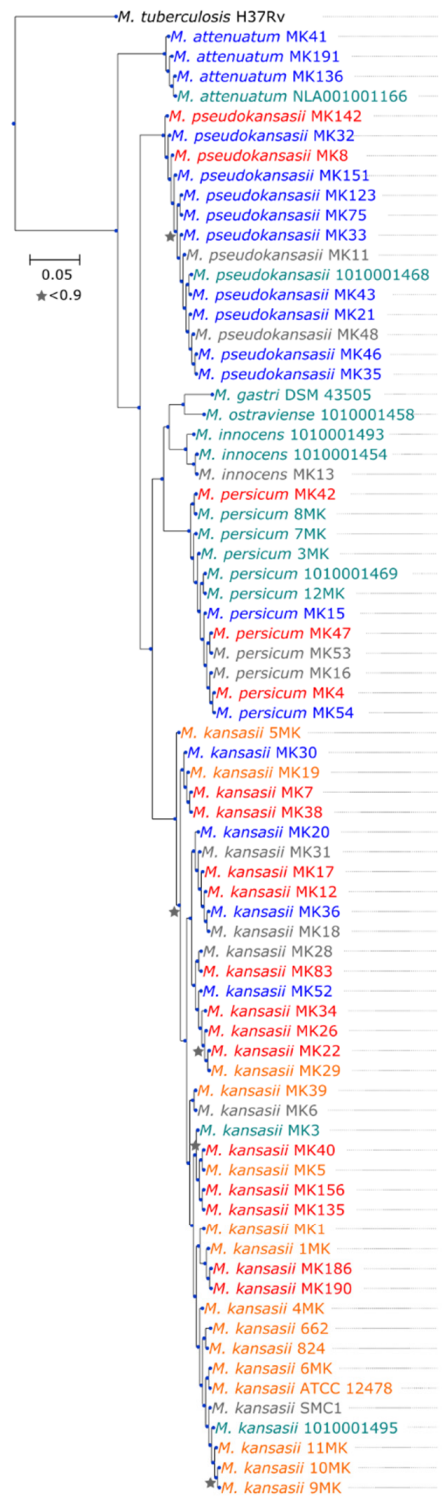

C

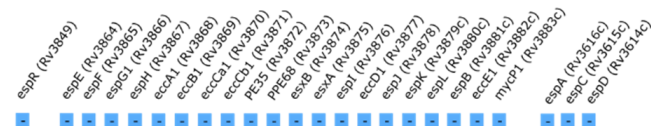

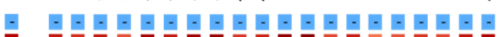

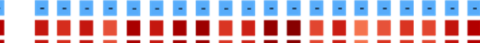

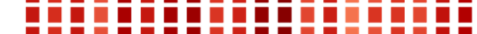

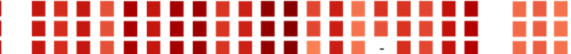

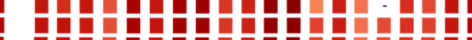

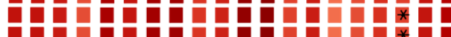

PryPrry

PCPPCH

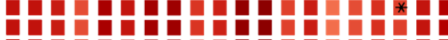

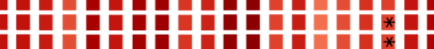

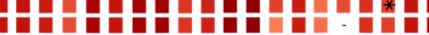

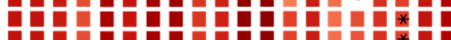

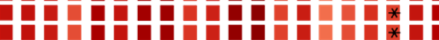

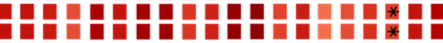

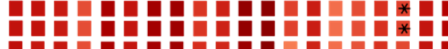

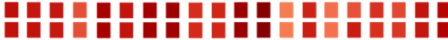

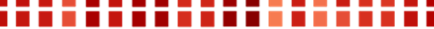

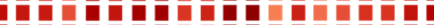

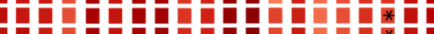

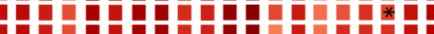

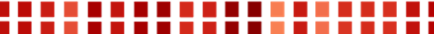

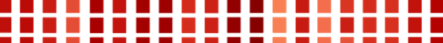

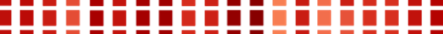

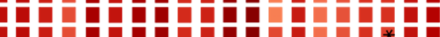

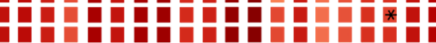

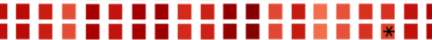

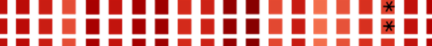

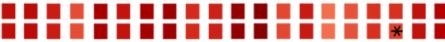

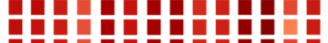

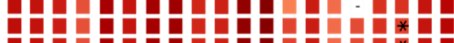

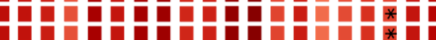

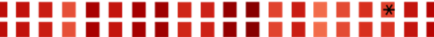

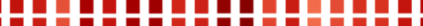

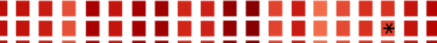

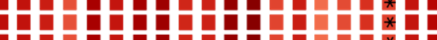

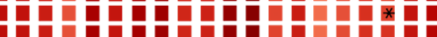

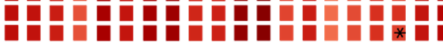

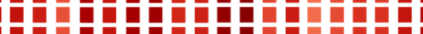

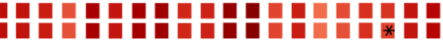

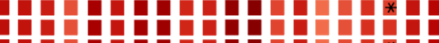

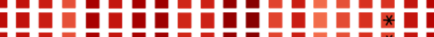

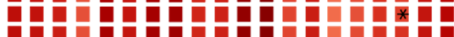

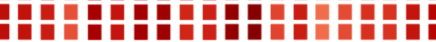

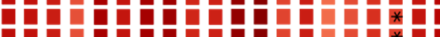

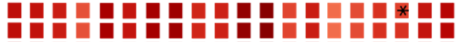

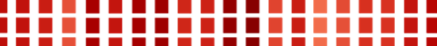

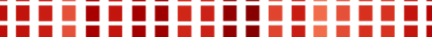

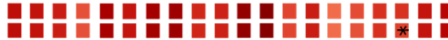

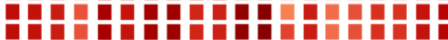

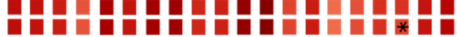

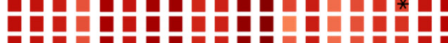

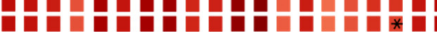

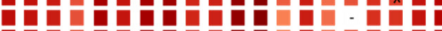

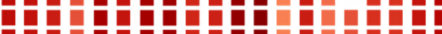

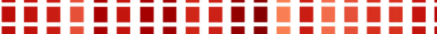

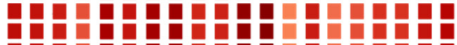

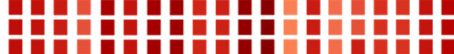

Amino acid identity

Figure 1. Maximum-likelihood core-genome phylogeny and conservation of the Type-VII secretion system-associated ESX-1 and esp $A B C$ loci among members of the M. kansasii complex (A) ESX-1 and EspACD loci of M. tuberculosis H37Rv. Ecc, ESX-1 conserved components; Esp, ESX-1 secreted protein. EsxA, ESAT-6; EsxB, CFP-10. EspACD locus is required for the secretion and function of EsxA and EsxB. (B) Maximum-likelihood phylogeny based on the concatenated amino acid alignments of single-copy orthologous proteins forming the core genome. Node supports based on the Shimodaira-Hasegawa (SH) test are indicated with a star when below 0.9. The scale bar represents the number of amino acid substitutions per site alongside the branches. Species pathogenicity is color-coded according to the results reported in Table 1 . Red $=1=$ pathogenic, Orange $=2=$ probably pathogenic, Grey $=3=$ unknown, Green $=4$ = probably non-pathogenic, Blue $=5=$ non-pathogenic. (C) Conservation of the type-VII secretion system ESX-1 locus and EspACD in members of the M. kansasii complex. EspACD was absent from all isolates of M. gastri, M. ostraviense, M. innocens and M. persicum. The amino acid identity with the reference M. tuberculosis sequence (in blue) is indicated. Stars in the column of espB homologs indicate that the gene was split due to assembly gaps. 


\subsection{Virulence Factors of the M. kansasii Complex}

The presence of homologs of known $M$. tuberculosis virulence factors of different categories was assessed: (1) ESX-1 locus (Figure 1A,C), (2) ESX-2/3/4/5 loci, (3) mycolic acid synthesis, (4) complex lipid synthesis, (5) other genes related to lipid synthesis, (6) catabolism of cholesterol, (7) cell envelope protein, mammalian cell entry operons, (8) lipoproteins, phagosomal arresting, (9) inhibition of apoptosis, (10) oxidative and nitrosative stress resistance, (11) proteases, (12) metal transporters, (13) PE/PPE family proteins, (14) two-component systems, (15) transcription factors and (16) sigma factors, as described in Forrellad et al. [56]. Most $M$. tuberculosis virulence factors were conserved in all genomes of the M. kansasii complex highlighting the close relatedness of these two clades (Data Sheet $1, M$. tuberculosis virulence factors used as query to look for orthologous and paralogous genes among the $M$. kansasii complex). In addition, all strains encoded at least five distinct type-VII secretion systems (additional secretion system loci were found on plasmid, cf. below). Although espB has previously been described to be lacking in some isolates of the M. kansasii complex [13], this was probably due to assembly gaps, and the gene itself may still be functional. By contrast, the esp $A C D$ operon was missing in M. persicum, M. ostraviense, M. innocens as well as in M. gastri (Figure 1C).

No orthogroup was found to be present only in pathogenic strains and absent from all non-pathogenic strains. Furthermore, no orthologous group was shared by all pathogenic strains and absent from all non-pathogenic strains of the individual species M. kansasii, M. persicum or M. pseudokansasii. Similarly, no orthologous group, InterPro domain or PFAM motif specific to $M$. kansasii strains was associated with tissue tropism, either for bone and joint infections or for urinary tract infections.

A total of 107 orthogroups were found to be specific and conserved in all M. kansasii strains. Forty-four of them only encoded hypothetical proteins, but many other groups could be related to virulence. Indeed, many genes were annotated as ESX-1 secretionassociated protein (Esp); EspE (10 orthologous groups), EspB (2 groups), EspF (1 group), EspL (1 group) and EspH (1 group). EspE and EspB homologs harbored WxG or LxxxD motifs known to be required for type-VII secretion (Figure S2) [62]. Both could be modelled using Phyre2 [63] and showed the typical helix-loop-helix structure of type-VII secretion effectors (Figure S2). In addition, four groups of PPE family proteins and one group of PE family proteins were likely substrates of the type-VII secretion system. Surprisingly, most of these homologs were located on a single genomic island present only in M. kansasii strains (Figure 2). In addition, this region encoded a homolog of EspG1, a chaperonin involved in the folding of effectors of the ESX-1 locus. This latter was not detected by the orthologous comparison because the orthologous group was shared by all strains; M. kansasii strains had two homologs, whereas other species of the complex only encoded one. Finally, one espK homolog, known to interact with EspB and EccCb1 (although not required for full virulence) [64], was also found in the mobile genetic element. Orthologs of EspK were also identified in M. pseudokansasii strains, but in a different genetic context, flanked with genes annotated as esp $B, \operatorname{es} x A, \operatorname{es} x B$ and espK. 


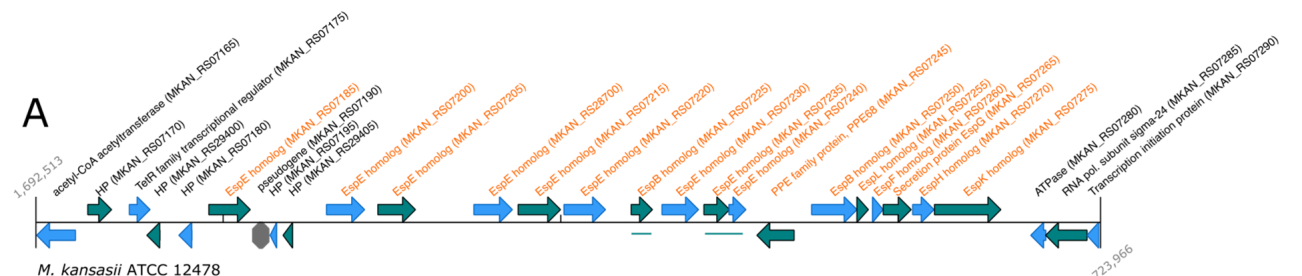

B
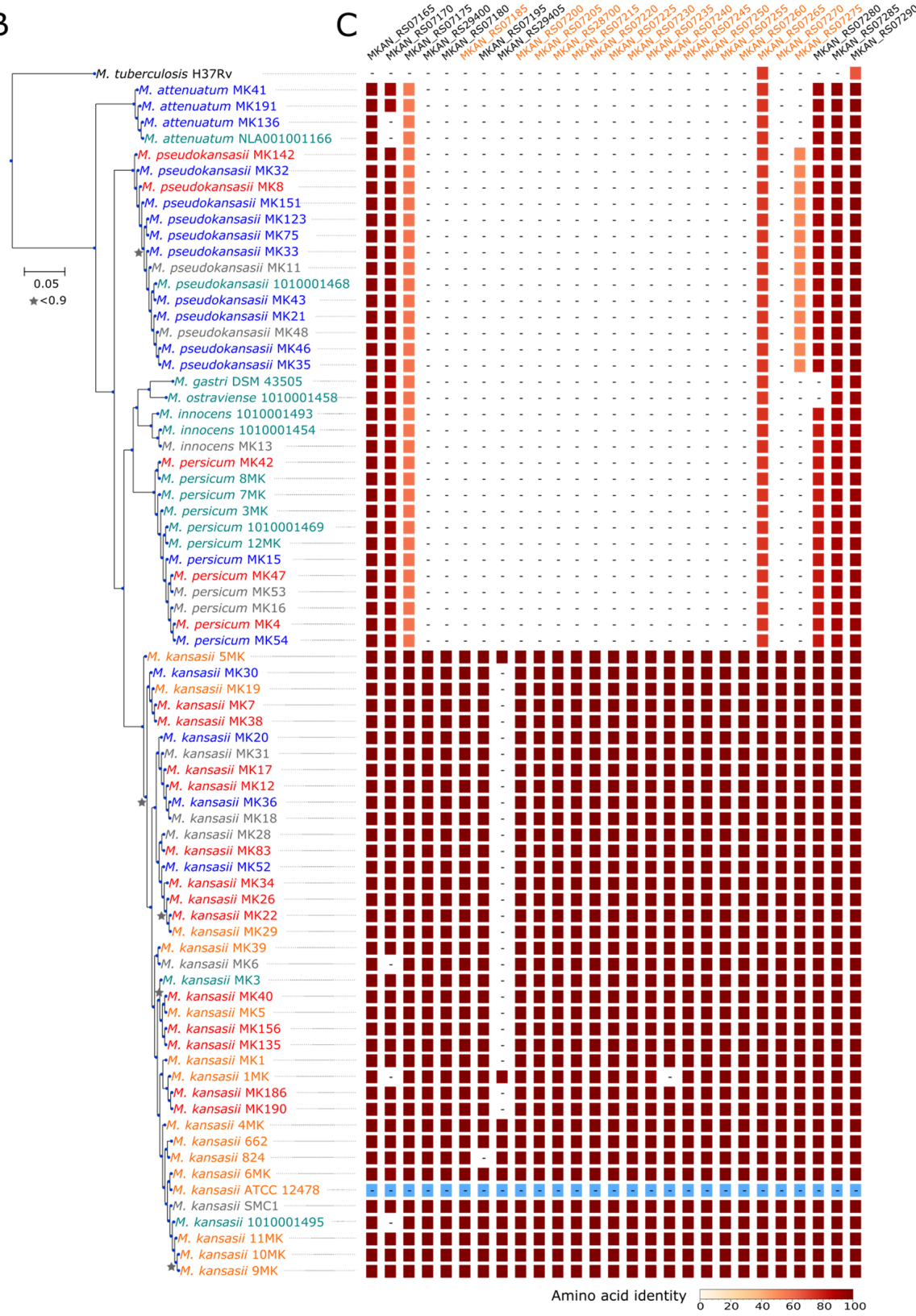

Figure 2. M. kansasii-specific genomic island encodes Type-VII secretion system effectors. (A) Locus is displayed for M. kansasii ATCC 12478, as a reference genome. Products mentioning "homolog" were manually annotated based on comparison to other genomes. Esp, ESX-1 secreted protein. All genes related to the type-VII secretion system are highlighted in orange. (B) Maximumlikelihood phylogeny based on the concatenated amino acid alignments of single-copy orthologous genes forming the core genome. Node supports based on the Shimodaira-Hasegawa (SH) test are indicated with a star when below 0.9. The scale bar represents the number of amino acid substitutions per site alongside the branches. Species pathogenicity is color-coded according to the results reported in Table 1 . Red $=1=$ pathogenic, Orange $=2=$ probably pathogenic, Grey $=3=$ unknown, Green $=4=$ probably non-pathogenic, Blue $=5=$ non-pathogenic. (C) The putative genomic island is specific to the M. kansasii species. The amino acid identity with the reference M. kansasii strain ATCC 12478 sequence (in blue) is color-coded. 


\subsection{Three Distributive Conjugal Transfers Induced Gene Losses Associated with Pathogenicity}

To investigate the existence of genetic determinants supporting increased virulence in M. kansasii complex, a genome-wide association study was performed using treeWAS [53]. This tool takes into account the phylogeny to score associations between phenotypic data (e.g., virulence level) and genetic variations (e.g., SNPs or presence/absence of genes). Three scores are computed by treeWAS: the terminal, the simultaneous and the subsequent scores. The terminal score compares and correlates leaves phenotypes and genotypes, regardless of the ancestral information. The simultaneous score is based on the number of branches with parallel changes (phenotype and genotypes). Finally, the subsequent score computes the proportion of the tree with simultaneous changes in genotypes and phenotypes.

The association between the presence/absence of single-copy orthogroups with pathogenic (categories 1 and 2) and non-pathogenic (categories 4 and 5) phenotypes in the complete dataset was significant for four and two orthogroups $(p<0.00001)$ with the simultaneous score and the terminal score, respectively (Figure 3A,B). Two orthogroups (group_5101 and group_5214) were identified by both scores: a hemerythrin-like protein and a nitroreductase-like protein that could be involved in the resistance to oxidative stresses. Both proteins are encoded by neighboring genes that could form an operon in M. kansasii (Figure 3C). The third significant orthogroup (group_5672) encoded a PE family protein and is likely a substrate of the type-VII secretion system. The last orthogroup (group_4940) encoded a hypothetical protein that was negatively associated with pathogenicity. However, the gene was pseudogenized in M. kansasii isolates and should hence not be considered (Figure S3). When assessing the association of single nucleotide polymorphisms (SNPs) present in the core-genome of M. kansasii strains with the phenotype, no signal could be detected.

Three of the four non-pathogenic M. kansasii isolates (MK20, MK30 and MK52) lacked the orthogroups identified by GWAS analysis (Figure S3). The region surrounding the hemerythrin-like and the nitroreductase-like proteins showed evidence of distributive conjugal transfer (DCT) originating from M. persicum for MK30 (25 kb) and MK52 (67 kb) and from M. attenuatum for MK20 (75 kb) (Figure 3D). This indicates that distinct recombination events in an overlapping genomic region led to the loss of the hemerythrin-like and nitroreductase-like proteins. The hemerythrin-like protein was absent in M. attenuatum and truncated (A4G31_RS07725; 65 aa) by a frameshift mutation in M. persicum (Figure 3C). Another nitroreductase-like protein identified in M. persicum clustered in a different orthogroup and was longer than the M. kansasii homolog and absent in M. attenuatum (Figure 3C).

Since DCT likely explained variations in M. kansasii pathogenicity, we wanted to further assess the occurrence of distributive conjugal transfers (DCT) between all the members of the $M$. kansasii complex. For this purpose, coding sequences showing a higher average amino acid identity with another species than its own were considered to be probable recombinant regions, as highlighted in Figure 4 for a subset of $M$. kansasii isolates (see Figures S4-S7 for the remaining M. kansasii isolates, M. persicum, M. pseudokansasii, and M. attenuatum, respectively). DCT signals were widespread in the M. kansasii complex, involving recombination of one gene up to large genomic fragments. Overall, $M$. kansasii presented larger regions of DCTs as compared with M. persicum, M. pseudokansasii and M. attenuatum. Conversely, several M. kansasii isolates presented fewer DCT signals in a similar fashion to the other species of the complex (Figure S4). In some cases, the signal of DCT is conserved in entire clades, suggesting that some DCTs likely occurred in common ancestors of these clades, and were conserved throughout further individual evolution of the strains. On the contrary, some DCTs were restricted to one isolate, indicating more recent transfer events. Although they might be widespread, this method is not designed to allow the detection of transfers between strains of the same species. Furthermore, DCTs from species that are not included cannot be detected. 


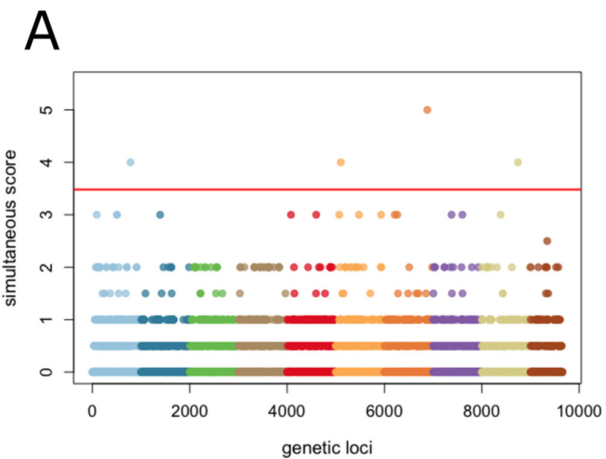

B

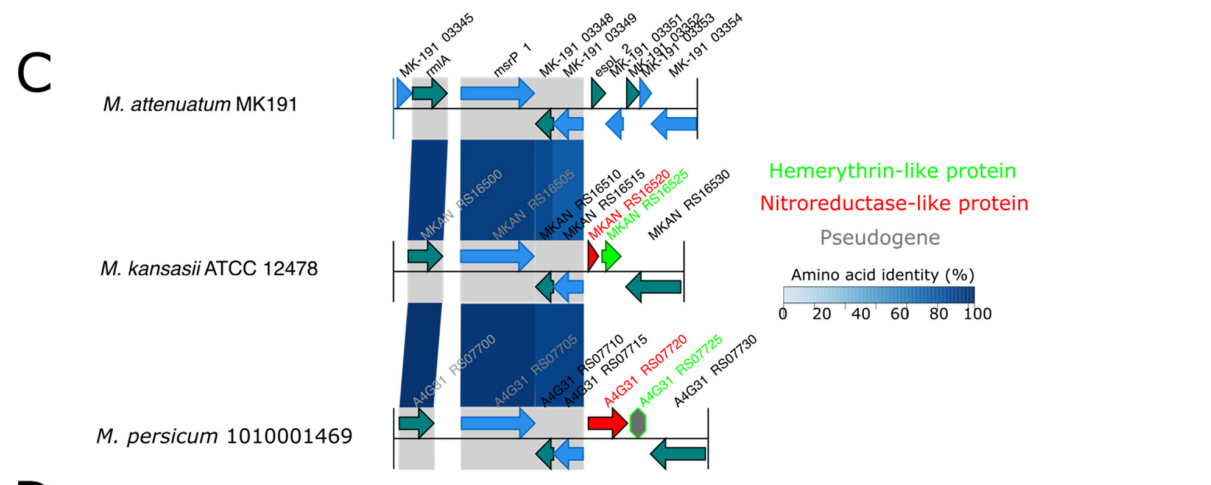

D

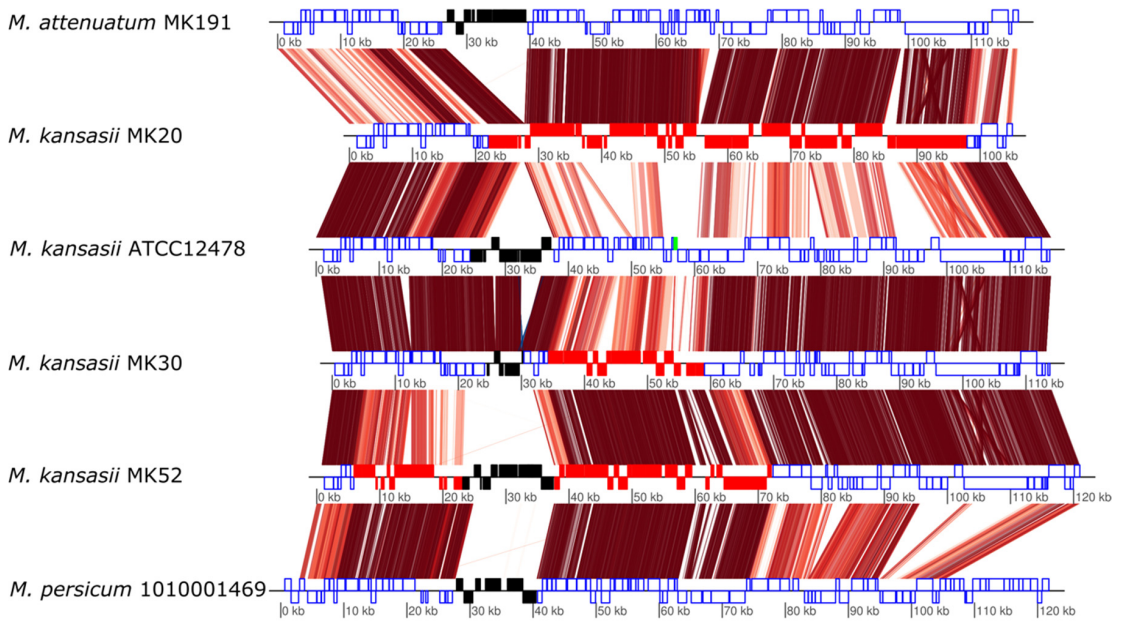

$20 \mathrm{~kb}$

Figure 3. treeWAS results based on a matrix of presence/absence of single-copy orthologs. (A) Manhattan plot (colors were randomly assigned) and (B) bar plot of the treeWAS simultaneous score based on the presence/absence of single-copy orthologs and the core-genome phylogeny. Group_5101 and group_5214 encode for a hemerythrin-like protein and a nitroreductase-like protein, respectively, that were also identified by the terminal score. Group_5672 encodes a PE-family protein that is likely a substrate of the type-VII secretion system. Group_4940 encodes for a hypothetical protein that was negatively associated with pathogenicity. However, the gene is pseudogenized in some M. kansasii isolates and is thus most probably non-functional. (C) Genomic context of group_5101 and group_5214 in the representative genomes of M. kansasii, M. persicum and M. attenuatum. The hemerythrin-like protein was absent and pseudogenized in M. attenuatum and M. persicum, respectively. The nitroreductase-like protein was longer in M. persicum as compared to M. kansasii while it was absent in M. attenuatum. (D) The extended genomic region surrounding the hemerythrin-like protein (highlighted in green in strain ATCC 12478) present distributive conjugal transfers between M. attenuatum and M. kansasii, as well as between M. persicum and M. kansasii, leading to the loss of this protein in MK20, MK30 and MK52. Amino acid identity (from TBLASTX searches) between genomes is represented by the red stripes with a color gradient from $80 \%$ (light red) to $100 \%$ (dark red). ORFs corresponding to prophage sequences were manually highlighted based on gene annotation (not detected using PHASTER). 


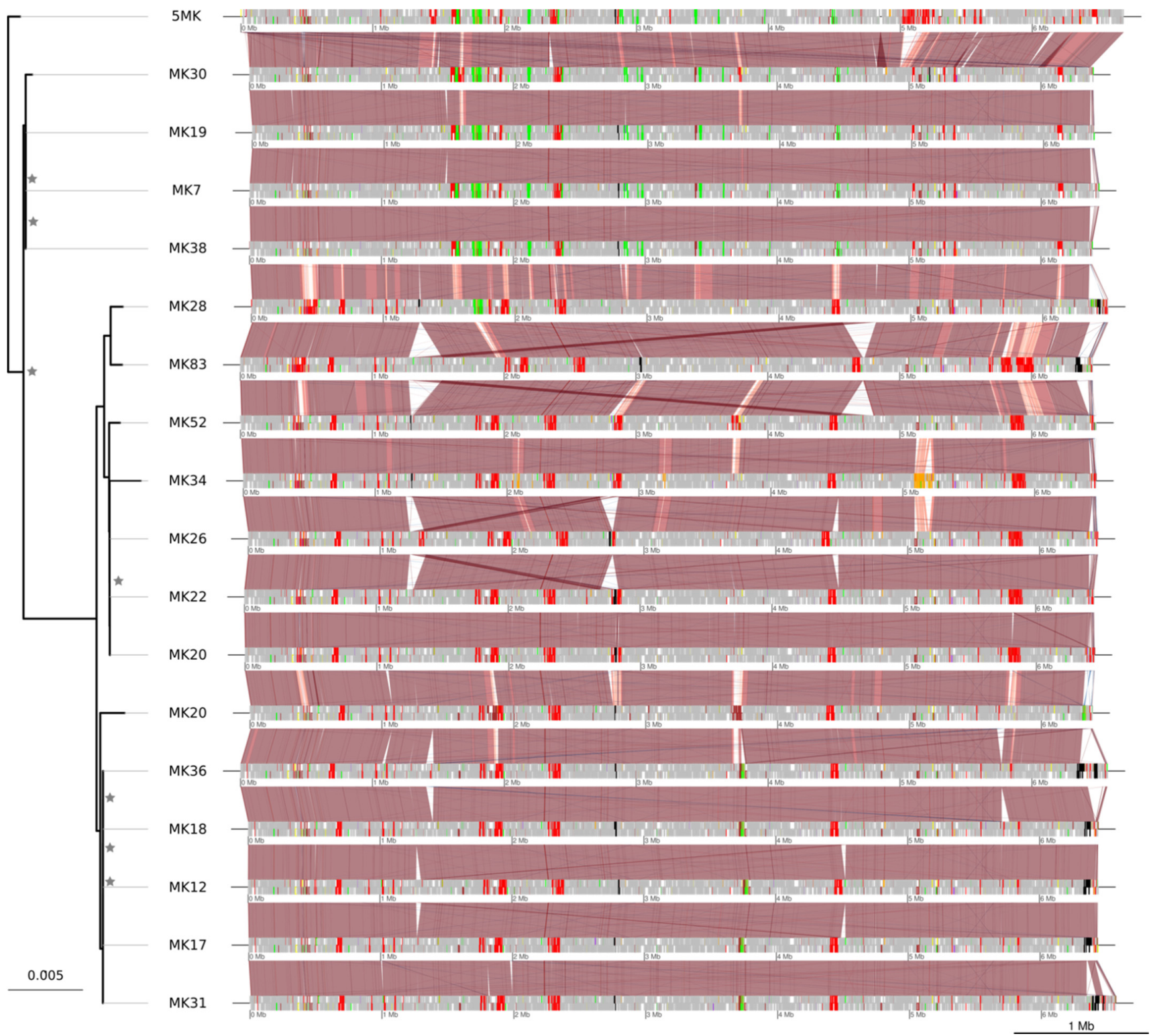

Figure 4. Distributive conjugal transfers within the species $M$. kansasii. For visual purposes, a subset of $M$. kansasii isolates was selected to illustrate the distributive conjugal transfers between members of the complex and the species $M$. kansasii. The remaining M. kansasii isolates are shown in Figure S4. The red stripes between genomes indicate aligned regions (BLASTN; $e$-value $<0.00001$, nucleotide identity $>87 \%$ ), with a color gradient indicating the identity from $87 \%$ (light red) to $100 \%$ (dark red). Recombining loci are highlighted in bright red when the recombination occurred with M. persicum, in green with M. pseudokansasii, in orange with M. ostraviense, in yellow with M. innocens, in brown with M. attenuatum and in purple with M. gastri. Predicted prophages are highlighted in black. Some large DCTs between M. persicum or M. pseudokansasii and M. kansasii likely occurred in ancestral strains since they can be observed in the whole subset of strains (absent in Figure S4). Conversely, other large DCT, such as that between M. innocens and M. kansasii MK34, are likely more recent. Although all genomes displayed in this figure are draft genomes, they exhibit a high collinearity after contig reordering. The maximum-likelihood phylogeny of the strains is an extracted subset of the core-genome phylogeny shown in Figure 1B. 


\subsection{Conjugative Plasmids Are Widespread but Not Associated with Pathogenicity}

Conjugative plasmids were found in 22 out of the 75 strains of the $M$. kansasii complex (29.3\%). Two conjugative plasmids were complete and circularized publicly available sequences (pMK12478 and pMK142). Interestingly, strain MK5 presented two different plasmids. All detected plasmids shared the same backbone structure as described by Ummels et al., although the approach used to identify plasmids likely biased their selection [31]. Only four plasmids lacked the traA/traI homolog but still shared type-VII and type-IV secretion systems homologs (Figure 5). As indicated by the high level of conservation ( $>99 \%$ of amino acid identities) between several plasmids as well as by the plasmid phylogeny, relatively recent conjugative plasmid transfers occurred between different isolates and different species of the M. kansasii complex (Figures S9 and S10). Sizes ranged from $60 \mathrm{Kbp}$ to $170 \mathrm{Kbp}$. The plasmid sizes may have been underestimated because some contigs could have been missed during the inclusion process despite the use of various backbone genes and GC-coverage plots. The mean GC content of plasmids was significantly lower than that of chromosomes ( $p$-value $<0.00001$, two sample $t$-test) (Figure $S 8$ ).

The presence of plasmids was not associated with variations in pathogenicity $(p$-value $=0.91$, Chi-square). Furthermore, the presence/absence of single-copy orthogroups was assessed previously with the treeWAS analysis and returned no plasmid-encoded hit. Conjugative plasmids exhibited various gene functions within and between plasmids as shown by the diversity of COG categories found (Figure S11). Interestingly, a type I-E CRISPR-cas system locus was found on the plasmid of M. pseudokansasii MK11 (Figure S12). Surprisingly, the best BLAST hits (using BLASTP) with the non-redundant protein database of the NCBI were found in the distantly related M. abscessus species. Such CRISPR-cas loci can be found on mobile genetic elements [65], but the transfer between a fast and a slow growing mycobacterium was unexpected.
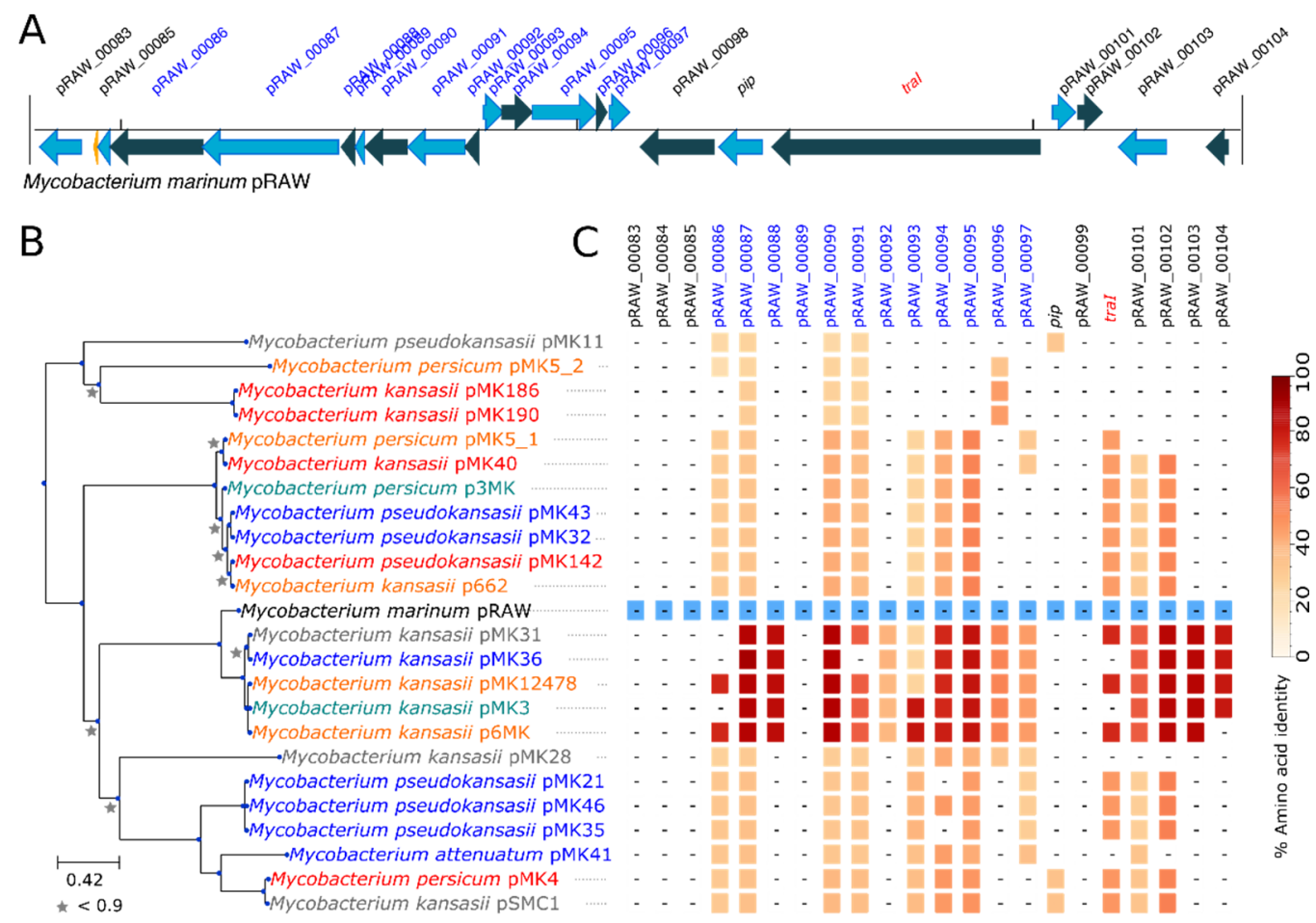

Figure 5. Cont. 


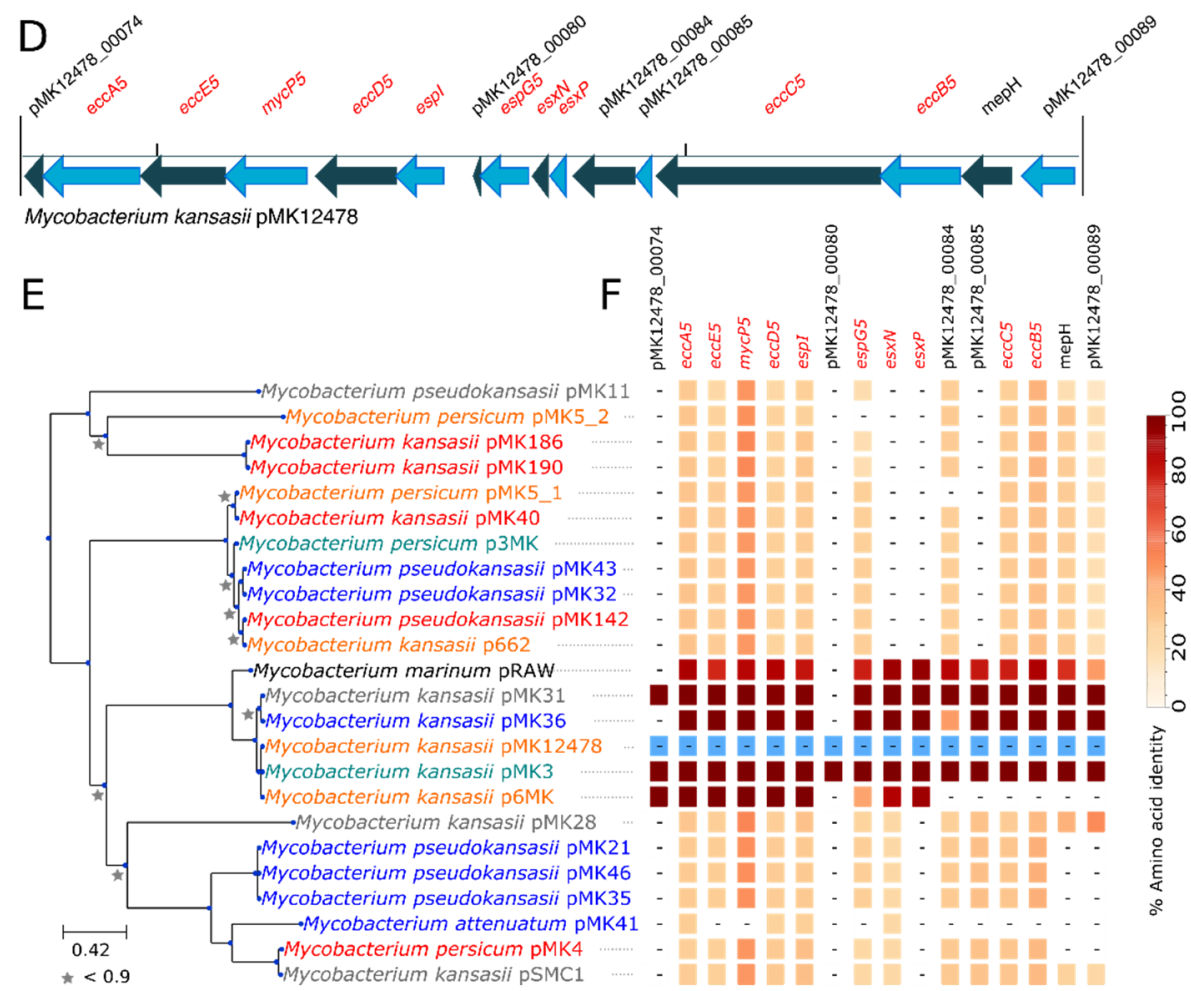

Figure 5. Distribution of the conjugative plasmids detected in the M. kansasii complex. (A) The type-IV secretion system locus (in blue), as well as the relaxase (traI) of the reference conjugative plasmid of M. marinum pRAW (as described in Ummels et al. [31]), is conserved (C) in some members of the M. kansasii complex. For multiple-copy orthologous groups, the amino acid identity of the best hit is shown. The reference sequences used to build this plot are indicated in blue. (D) Schematic representation of the plasmid-encoded Type-VII secretion system of strain ATCC 12478 and its gene conservation (F) in members of the M. kansasii complex. (B,E) Maximum-likelihood phylogeny based on the concatenated alignment of six single-copy orthologous genes (2766 amino acids) of the plasmid dataset. Bootstrap values below 0.9 are indicated with grey stars. The colors of the labels indicate pathogenicity, as reported in Table 1 . Red $=1=$ pathogenic, Orange $=2=$ probably pathogenic, Grey $=3=$ unknown, Green $=4=$ probably non-pathogenic, Blue $=5=$ non-pathogenic. Closely related plasmids sharing $>99 \%$ of amino acid identities were found in different species, indicating probable recent conjugative transfers between species of the complex.

\section{Discussion}

The M. kansasii complex currently regroups seven closely related species sharing nearly $90 \%$ average nucleotide identity. Their prevalence and pathogenicity vary significantly [11,66-69], but as observed in our dataset as well, M. kansasii is the most frequently isolated and most pathogenic species. The unexpected isolation of two M. pseudokansasii strains in urine specimens could suggest a particular tropism in this species, which remains to be confirmed in larger studies. The comparative genomic analysis revealed several elements that may contribute to the differences in pathogenicity between members of the M. kansasii complex. First, M. persicum, $M$. ostraviense, $M$. innocens and $M$. gastri lacked the operon encoding for EspACD, which are major virulence factors of $M$. tuberculosis [70]. Indeed, in M. tuberculosis, the secretion of ESAT-6 and CFP-10 effectors requires the expression of EspA $[19,70]$ and maintenance of wild-type levels of EspA require both EspC and EspD [71]. Recently, it has even been postulated that EspC could act as the needle of the type-VII secretion system apparatus [72]. Thus, in light of the conservation of other known virulence factors, the absence of the espACD operon could have a role in the reduced pathogenicity of these species and in their rare isolation from patients. However, both M. pseudokansasii and M. attenuatum - two mostly non-pathogenic species—also encoded this locus, and hence additional genetic factors may explain the increased virulence of M. kansasii. 
Going along with this hypothesis, $M$. kansasii harbored a large genomic island, encoding 17 effectors of the type-VII secretion system that could play a role in the virulence and the interaction of M. kansasii strains with its host. Although M. pseudokansasii exhibited another genomic region encoding putative type-VII secretion system effectors, it was much shorter (1 espK, 1 espB and 2 WxG-100-familly proteins homologs). Further studies using mutant and wild type strains will be needed to investigate the role of these genetic factors in the pathogenicity of the M. kansasii complex.

In $M$. tuberculosis, variation in virulence were seen after functional modifications of master regulators of virulence, such as the PhoPR or the DosR regulon [56,73]. However, all regulators investigated (Data sheet 1) shared the same amino acid identities within species and no single nucleotide polymorphism (SNPs) of the M. kansasii species was significantly associated with pathogenicity. However, two genes, encoding hemerythrin-like and nitroreductase-like proteins, were significantly associated with pathogenic phenotypes (Figure 3). In M. tuberculosis, the hemerythrin-like protein Rv2633c is one of the genes that are up-regulated upon infection into THP-1 macrophages [74,75]. Surprisingly, Rv2633c was recently shown not to bind $\mathrm{O}_{2}$, as could have been expected from a hemerythrin, but exhibited a catalase activity [76]. Although the M. kansasii hemerythrin-like protein did not cluster with the same orthogroup as Rv2633c, it could play a similar role as it is located upstream a nitroreductase-like protein, and genes encoded in operon frequently harbor related functions [77]. Since protection against reactive oxygen and reactive nitrogen species are critical for the intracellular survival in macrophages, we hypothesize that the loss of these genes could cause a decreased virulence.

Among possible evolutionary processes that could lead to hemerythrin and nitroreductase gene loss, DCTs represent the most likely mechanism for the following reasons. The large region affected by transfer precludes classical transformation mechanisms that usually involve the transfer of single small DNA fragments $(3-5 \mathrm{~Kb})$ [28]. Indeed, DCT can mobilize several DNA fragments of up to $200 \mathrm{~Kb}$, creating mosaic genomes through recombination [28,35]. M. kansasii strains MK30 and MK52 likely received multiple DCTs from M. persicum (in red in Figure 4) whereas M. kansasii MK20 harbored several large recombining regions from $M$. attenuatum (in brown in Figure 4). Moreover, these regions were not prophages based on a manual inspection of the annotation and on PHASTER predictions, nor integrated chromosomal conjugative plasmids (episomes), ruling out transduction and classical conjugation, respectively.

The similarity of conjugative plasmids across different species of the M. kansasii complex suggests the occurrence of HGTs most likely through conjugation among and within species. This is corroborated by the phylogenetic reconstruction based on coreplasmid genes that intertwines plasmids belonging to different species in the same clades. In the present dataset, the pathogenicity of the strains could not be correlated with the presence or absence of plasmids, or some of their genetic content, but we cannot exclude an eventual role in bacterial pathogenicity. Indeed, the incomplete nature of genome assemblies often renders difficult the identification of all contigs forming the plasmids, and the method used here could have biased our analysis towards the identification of previously known conjugative plasmids.

Overall, comparative analyses and genome-wide association studies on 74 genomes could identify several loci likely associated with the increased or decreased pathogenicity in species of the M. kansasii complex, as well as among M. kansasii strains. DCTs, conjugative plasmid transfers and transduction were frequently observed and several evidence indicate that DCT was directly involved in gene losses affecting strain pathogenicity. While DCT was thought to be an important mechanism for HGT in mycobacteria [28], it has, to our knowledge, never previously been directly associated with a decreased pathogenicity. This demonstrates the previously unsuspected importance of HGT between and within species, conferring a high level of genome plasticity to these non-tuberculous mycobacteria, and likely providing a selective advantage and faster genome evolution. The existence and 
the extent of such genetic transfers with other mycobacteria remain to be investigated by including further distantly related species in similar comparative analyses.

Supplementary Materials: The following are available online at https:/ /www.mdpi.com/2076-260 7/9/2/348/s1, Figure S1: Core-genome phylogeny and genomic features; Figure S2: espE homologs of M. kansasii located on the genomic island described in Figure 2; Figure S3: Distribution of the homologs of the best treeWAS hits; Figure S4: Distributive conjugal transfer events among the M. kansasii species; Figure S5: Distributive conjugal transfer events among the M. persicum species; Figure S6: Distributive conjugal transfer events among the M. pseudokansasii species; Figure S7: Distributive conjugal transfer events among the M. attenuatum species; Figure S8: Genomic features of the conjugative plasmids; Figure S9: Alignment of the complete plasmid sequence of $M$. kansasii strain ATCC 12478 and four closely related plasmids of the dataset; Figure S10: Alignment of the complete plasmid sequence of M. pseudokansasii strain MK142 and six closely related plasmids of the dataset; Figure S11: Cluster of Orthologous Genes (COG) annotated on the identified conjugative plasmids; Figure S12: Plasmid-encoded CRISPR-cas system; Data sheet 1: M. tuberculosis virulence factors used as query to look for orthologous and paralogous genes among the M. kansasii complex [78-80].

Author Contributions: F.T. designed the study, performed the bioinformatic analyses and wrote the manuscript. T.P. and C.B. helped with the bioinformatic analyses and corrected the manuscript. K.J. provided the strains of the study and corrected the manuscript. G.G. directed and designed the study, and corrected the manuscript. All authors have read and agreed to the published version of the manuscript.

Funding: This research received no external funding.

Institutional Review Board Statement: All procedures performed in this study involving human participants were carried out in accordance with the ethical standards of the regional (Project ID: 2017-00194, accepted on the 01.03.2017 by the regional ethical committee) and national research committee and with the 1964 Helsinki declaration and its later amendments or comparable ethical standards.

Informed Consent Statement: Patient consent was waived due to (i) the fact that a significant proportion of patients had already died, (ii) most strains were isolated several years prior to the study and many patients moved and/or were not followed up in our institution anymore, (iii) our study included only limited retrospective data and there was no intervention on patient or foreseen potential negative impact on them (iv) sequencing was performed on pure culture and therefore we gathered no DNA sequence from the host, (v) there was no record of patient opposition to reuse data at our disposal for the patient included in the study.

Data Availability Statement: All sequencing data are available under the BioProject accession numbers PRJEB25525 and PRJEB28857.

Acknowledgments: We thank Sébastien Aeby, who did the sequencing work. We also thank Nicolas Troillet for providing some clinical data of patients from Valais canton. The computations were performed at the Vital-IT Center (http: / / www.vital-it.ch) for high-performance computing of the SIB Swiss Institute of Bioinformatics.

Conflicts of Interest: The authors declare no conflict of interest.

\section{References}

1. Field, S.K.; Cowie, R.L. Lung Disease Due to the More Common Nontuberculous Mycobacteria. Chest 2006, $129,1653-1672$. [CrossRef]

2. Prevots, D.R.; Marras, T.K. Epidemiology of Human Pulmonary Infection with Nontuberculous Mycobacteria: A Review. Clin. Chest Med. 2015, 36, 13-34. [CrossRef]

3. Griffith, D.E.; Aksamit, T.; Brown-Elliott, B.A.; Catanzaro, A.; Daley, C.; Gordin, F.; Holland, S.M.; Horsburgh, R.; Huitt, G.; Iademarco, M.F.; et al. An Official ATS/IDSA Statement: Diagnosis, Treatment, and Prevention of Nontuberculous Mycobacterial Diseases. Am. J. Respir. Crit. Care Med. 2007, 175, 367-416. [CrossRef]

4. Bhatt, K.; Banavathi, K. Mycobacterium Kansasii Osteomyelitis-A Masquerading Disease. JMM Case Rep. 2018,5 , e005114. [CrossRef] 
5. Alvarado-Esquivel, C.; García-Corral, N.; Carrero-Dominguez, D.; Enciso-Moreno, J.A.; Gurrola-Morales, T.; Portillo-Gómez, L.; Rossau, R.; Mijs, W. Molecular Analysis of Mycobacterium Isolates from Extrapulmonary Specimens Obtained from Patients in Mexico. BMC Clin. Pathol. 2009, 9, 1. [CrossRef]

6. Brooker, W.J.; Aufderheide, A.C. Genitourinary Tract Infections Due to Atypical Mycobacteria. J. Urol. 1980, 124, $242-244$. [CrossRef]

7. Alcaide, F.; Richter, I.; Bernasconi, C.; Springer, B.; Hagenau, C.; Schulze-Röbbecke, R.; Tortoli, E.; Martín, R.; Böttger, E.C.; Telenti, A. Heterogeneity and Clonality among Isolates of Mycobacterium Kansasii: Implications for Epidemiological and Pathogenicity Studies. J. Clin. Microbiol. 1997, 35, 1959-1964. [CrossRef]

8. Devallois, A.; Goh, K.S.; Rastogi, N. Rapid Identification of Mycobacteria to Species Level by PCR-Restriction Fragment Length Polymorphism Analysis of the Hsp65 Gene and Proposition of an Algorithm to Differentiate 34 Mycobacterial Species. J. Clin. Microbiol. 1997, 35, 2969-2973. [CrossRef]

9. Bakuła, Z.; Modrzejewska, M.; Safianowska, A.; van Ingen, J.; Proboszcz, M.; Bielecki, J.; Jagielski, T. Proposal of a New Method for Subtyping of Mycobacterium Kansasii Based upon PCR Restriction Enzyme Analysis of the Tuf Gene. Diagn. Microbiol. Infect. Dis. 2016, 84, 318-321. [CrossRef]

10. Tagini, F.; Aeby, S.; Bertelli, C.; Droz, S.; Casanova, C.; Prod'hom, G.; Jaton, K.; Greub, G. Phylogenomics Reveal That Mycobacterium Kansasii Subtypes Are Species-Level Lineages. Description of Mycobacterium Pseudokansasii Sp. Nov., Mycobacterium Innocens Sp. Nov. and Mycobacterium Attenuatum Sp. Nov. Int. J. Syst. Evol. Microbiol. 2019, 69, 1696-1704. [CrossRef]

11. Taillard, C.; Greub, G.; Weber, R.; Pfyffer, G.E.; Bodmer, T.; Zimmerli, S.; Frei, R.; Bassetti, S.; Rohner, P.; Piffaretti, J.-C.; et al. Clinical Implications of Mycobacterium Kansasii Species Heterogeneity: Swiss National Survey. J. Clin. Microbiol. 2003, 41, 1240-1244. [CrossRef]

12. Shahraki, A.H.; Trovato, A.; Mirsaeidi, M.; Borroni, E.; Heidarieh, P.; Hashemzadeh, M.; Shahbazi, N.; Cirillo, D.M.; Tortoli, E. Mycobacterium Persicum Sp. Nov., a Novel Species Closely Related to Mycobacterium Kansasii and Mycobacterium Gastri. Int. J. Syst. Evol. Microbiol. 2017, 67, 1766-1770. [CrossRef] [PubMed]

13. Jagielski, T.; Borówka, P.; Bakuła, Z.; Lach, J.; Marciniak, B.; Brzostek, A.; Dziadek, J.; Dziurzyński, M.; Pennings, L.; van Ingen, J.; et al. Genomic Insights Into the Mycobacterium Kansasii Complex: An Update. Front. Microbiol. 2020, $10,2918$. [CrossRef] [PubMed]

14. Wang, J.; McIntosh, F.; Radomski, N.; Dewar, K.; Simeone, R.; Enninga, J.; Brosch, R.; Rocha, E.P.; Veyrier, F.J.; Behr, M.A. Insights on the Emergence of Mycobacterium Tuberculosis from the Analysis of Mycobacterium Kansasii. Genome Biol. Evol. 2015, 7, 856-870. [CrossRef]

15. Gröschel, M.I.; Sayes, F.; Simeone, R.; Majlessi, L.; Brosch, R. ESX Secretion Systems: Mycobacterial Evolution to Counter Host Immunity. Nat. Rev. Microbiol. 2016, 14, 677-691. [CrossRef]

16. Ma, Y.; Keil, V.; Sun, J. Characterization of Mycobacterium Tuberculosis EsxA Membrane Insertion: Roles of N- and C-Terminal Flexible Arms and Central Helix-Turn-Helix Motif. J. Biol. Chem. 2015, 290, 7314-7322. [CrossRef]

17. Simeone, R.; Bobard, A.; Lippmann, J.; Bitter, W.; Majlessi, L.; Brosch, R.; Enninga, J. Phagosomal Rupture by Mycobacterium Tuberculosis Results in Toxicity and Host Cell Death. PLoS Pathog. 2012, 8, e1002507. [CrossRef] [PubMed]

18. Majlessi, L.; Prados-Rosales, R.; Casadevall, A.; Brosch, R. Release of Mycobacterial Antigens. Immunol. Rev. 2015, $264,25-45$. [CrossRef]

19. Fortune, S.M.; Jaeger, A.; Sarracino, D.A.; Chase, M.R.; Sassetti, C.M.; Sherman, D.R.; Bloom, B.R.; Rubin, E.J. Mutually Dependent Secretion of Proteins Required for Mycobacterial Virulence. Proc. Natl. Acad. Sci. USA 2005, 102, 10676-10681. [CrossRef]

20. Houben, D.; Demangel, C.; van Ingen, J.; Perez, J.; Baldeón, L.; Abdallah, A.M.; Caleechurn, L.; Bottai, D.; van Zon, M.; de Punder, K.; et al. ESX-1-Mediated Translocation to the Cytosol Controls Virulence of Mycobacteria. Cell. Microbiol. 2012, 14, 1287-1298. [CrossRef]

21. Arend, S.M.; de Haas, P.; Leyten, E.; Rosenkrands, I.; Rigouts, L.; Andersen, P.; Mijs, W.; van Dissel, J.T.; van Soolingen, D. ESAT-6 and CFP-10 in Clinical versus Environmental Isolates of Mycobacterium Kansasii. J. Infect. Dis. 2005, 191, 1301-1310. [CrossRef]

22. Goy, G.; Thomas, V.; Rimann, K.; Jaton, K.; Prod'hom, G.; Greub, G. The Neff Strain of Acanthamoeba Castellanii, a Tool for Testing the Virulence of Mycobacterium Kansasii. Res. Microbiol. 2007, 158, 393-397. [CrossRef]

23. Soucy, S.M.; Huang, J.; Gogarten, J.P. Horizontal Gene Transfer: Building the Web of Life. Nat. Rev. Genet. 2015, 16, 472-482. [CrossRef]

24. Gagneux, S. Ecology and Evolution of Mycobacterium Tuberculosis. Nat. Rev. Microbiol. 2018, 16, 202-213. [CrossRef]

25. Boritsch, E.C.; Khanna, V.; Pawlik, A.; Honoré, N.; Navas, V.H.; Ma, L.; Bouchier, C.; Seemann, T.; Supply, P.; Stinear, T.P.; et al. Key Experimental Evidence of Chromosomal DNA Transfer among Selected Tuberculosis-Causing Mycobacteria. Proc. Natl. Acad. Sci. USA 2016, 113, 9876-9881. [CrossRef]

26. Tsukamura, M.; Hasimoto, M.; Noda, Y. Transformation of Isoniazid and Streptomycin Resistance in Mycobacterium Avium by the Desoxyribonucleate Derived from Isoniazid- and Streptomycin-Double-Resistant Cultures. Am. Rev. Respir. Dis. 1960, 81, 403-406. [CrossRef]

27. Norgard, M.V.; Imaeda, T. Physiological Factors Involved in the Transformation of Mycobacterium Smegmatis. J. Bacteriol. 1978, 133, 1254-1262. [CrossRef]

28. Gray, T.A.; Derbyshire, K.M. Blending Genomes: Distributive Conjugal Transfer in Mycobacteria, a Sexier Form of HGT. Mol. Microbiol. 2018, 108, 601-613. [CrossRef] 
29. Hatfull, G.F.; Science Education Alliance Phage Hunters Advancing Genomics and Evolutionary Science Program; KwaZuluNatal Research Institute for Tuberculosis and HIV Mycobacterial Genetics Course Students; Phage Hunters Integrating Research and Education Program. Complete Genome Sequences of 138 Mycobacteriophages. J. Virol. 2012, 86, 2382-2384. [CrossRef]

30. Basra, S.; Anany, H.; Brovko, L.; Kropinski, A.M.; Griffiths, M.W. Isolation and Characterization of a Novel Bacteriophage against Mycobacteriumavium Subspecies Paratuberculosis. Arch. Virol. 2014, 159, 2659-2674. [CrossRef]

31. Ummels, R.; Abdallah, A.M.; Kuiper, V.; Aâjoud, A.; Sparrius, M.; Naeem, R.; Spaink, H.P.; van Soolingen, D.; Pain, A.; Bitter, W. Identification of a Novel Conjugative Plasmid in Mycobacteria That Requires Both Type IV and Type VII Secretion. mBio 2014, 5, e01744-14. [CrossRef] [PubMed]

32. da Rabello, M.C.S.; Matsumoto, C.K.; de Almeida, L.G.P.; Menendez, M.C.; de Oliveira, R.S.; Silva, R.M.; Garcia, M.J.; Leão, S.C. First Description of Natural and Experimental Conjugation between Mycobacteria Mediated by a Linear Plasmid. PLoS ONE 2012, 7, e29884. [CrossRef]

33. Stinear, T.P.; Pryor, M.J.; Porter, J.L.; Cole, S.T. Functional Analysis and Annotation of the Virulence Plasmid PMUM001 from Mycobacterium Ulcerans. Microbiology 2005, 151, 683-692. [CrossRef]

34. Mizuguchi, Y.; Suga, K.; Tokunaga, T. Multiple Mating Types of Mycobacterium Smegmatis. Jpn. J. Microbiol. 1976, 20, 435-443. [CrossRef] [PubMed]

35. Gray, T.A.; Krywy, J.A.; Harold, J.; Palumbo, M.J.; Derbyshire, K.M. Distributive Conjugal Transfer in Mycobacteria Generates Progeny with Meiotic-like Genome-Wide Mosaicism, Allowing Mapping of a Mating Identity Locus. PLoS Biol. 2013, 11, e1001602. [CrossRef]

36. Wang, J.; Karnati, P.K.; Takacs, C.M.; Kowalski, J.C.; Derbyshire, K.M. Chromosomal DNA Transfer in Mycobacterium Smegmatis Is Mechanistically Different from Classical Hfr Chromosomal DNA Transfer. Mol. Microbiol. 2005, 58, 280-288. [CrossRef]

37. Campbell, I. Chi-Squared and Fisher-Irwin Tests of Two-by-Two Tables with Small Sample Recommendations. Stat. Med. 2007, 26,3661-3675. [CrossRef]

38. Diagnosis and Treatment of Disease Caused by Nontuberculous Mycobacteria. Am. J. Respir. Crit. Care Med. 1997, 156, S1-S25. [CrossRef] [PubMed]

39. Bolger, A.M.; Lohse, M.; Usadel, B. Trimmomatic: A Flexible Trimmer for Illumina Sequence Data. Bioinformatics 2014, 30, 2114-2120. [CrossRef] [PubMed]

40. Bankevich, A.; Nurk, S.; Antipov, D.; Gurevich, A.A.; Dvorkin, M.; Kulikov, A.S.; Lesin, V.M.; Nikolenko, S.I.; Pham, S.; Prjibelski, A.D.; et al. SPAdes: A New Genome Assembly Algorithm and Its Applications to Single-Cell Sequencing. J. Comput. Biol. 2012, 19, 455-477. [CrossRef] [PubMed]

41. Rissman, A.I.; Mau, B.; Biehl, B.S.; Darling, A.E.; Glasner, J.D.; Perna, N.T. Reordering Contigs of Draft Genomes Using the Mauve Aligner. Bioinformatics 2009, 25, 2071-2073. [CrossRef] [PubMed]

42. Seemann, T. Prokka: Rapid Prokaryotic Genome Annotation. Bioinformatics 2014, 30, 2068-2069. [CrossRef] [PubMed]

43. Tagini, F.; Pillonel, T.; Croxatto, A.; Bertelli, C.; Koutsokera, A.; Lovis, A.; Greub, G. Distinct Genomic Features Characterize Two Clades of Corynebacterium Diphtheriae: Proposal of Corynebacterium Diphtheriae Subsp. Diphtheriae Subsp. Nov. and Corynebacterium Diphtheriae Subsp. Lausannense Subsp. Nov. Front. Microbiol. 2018, 9, 1743. [CrossRef]

44. Altschul, S.F.; Madden, T.L.; Schäffer, A.A.; Zhang, J.; Zhang, Z.; Miller, W.; Lipman, D.J. Gapped BLAST and PSI-BLAST: A New Generation of Protein Database Search Programs. Nucleic Acids Res. 1997, 25, 3389-3402. [CrossRef]

45. Galperin, M.Y.; Makarova, K.S.; Wolf, Y.I.; Koonin, E.V. Expanded Microbial Genome Coverage and Improved Protein Family Annotation in the COG Database. Nucleic Acids Res. 2015, 43, D261-D269. [CrossRef]

46. Kanehisa, M.; Sato, Y.; Morishima, K. BlastKOALA and GhostKOALA: KEGG Tools for Functional Characterization of Genome and Metagenome Sequences. J. Mol. Biol. 2016, 428, 726-731. [CrossRef] [PubMed]

47. Jones, P.; Binns, D.; Chang, H.-Y.; Fraser, M.; Li, W.; McAnulla, C.; McWilliam, H.; Maslen, J.; Mitchell, A.; Nuka, G.; et al. InterProScan 5: Genome-Scale Protein Function Classification. Bioinformatics 2014, 30, 1236-1240. [CrossRef]

48. Emms, D.M.; Kelly, S. OrthoFinder: Solving Fundamental Biases in Whole Genome Comparisons Dramatically Improves Orthogroup Inference Accuracy. Genome Biol. 2015, 16, 157. [CrossRef]

49. Katoh, K.; Standley, D.M. MAFFT Multiple Sequence Alignment Software Version 7: Improvements in Performance and Usability. Mol. Biol. Evol. 2013, 30, 772-780. [CrossRef]

50. Stamatakis, A. RAxML Version 8: A Tool for Phylogenetic Analysis and Post-Analysis of Large Phylogenies. Bioinformatics 2014, 30, 1312-1313. [CrossRef]

51. Mao, X.; Ma, Q.; Zhou, C.; Chen, X.; Zhang, H.; Yang, J.; Mao, F.; Lai, W.; Xu, Y. DOOR 2.0: Presenting Operons and Their Functions through Dynamic and Integrated Views. Nucleic Acids Res. 2014, 42, D654-D659. [CrossRef]

52. Price, M.N.; Dehal, P.S.; Arkin, A.P. FastTree 2-Approximately Maximum-Likelihood Trees for Large Alignments. PLoS ONE 2010, 5, e9490. [CrossRef]

53. Collins, C.; Didelot, X. A Phylogenetic Method to Perform Genome-Wide Association Studies in Microbes That Accounts for Population Structure and Recombination. PLoS Comput. Biol. 2018, 14, e1005958. [CrossRef]

54. Treangen, T.J.; Ondov, B.D.; Koren, S.; Phillippy, A.M. The Harvest Suite for Rapid Core-Genome Alignment and Visualization of Thousands of Intraspecific Microbial Genomes. Genome Biol. 2014, 15, 524. [CrossRef] [PubMed]

55. Seemann, T. Snippy: Fast Bacterial Variant Calling from NGS Reads. Available online: https://github.com/tseemann/snippy (accessed on 8 February 2021). 
56. Forrellad, M.A.; Klepp, L.I.; Gioffré, A.; Sabio y García, J.; Morbidoni, H.R.; de la Paz Santangelo, M.; Cataldi, A.A.; Bigi, F. Virulence Factors of the Mycobacterium Tuberculosis Complex. Virulence 2013, 4, 3-66. [CrossRef] [PubMed]

57. Simeone, R.; Bottai, D.; Frigui, W.; Majlessi, L.; Brosch, R. ESX/Type VII Secretion Systems of Mycobacteria: Insights into Evolution, Pathogenicity and Protection. Tuberculosis 2015, 95 (Suppl. 1), S150-S154. [CrossRef] [PubMed]

58. Arndt, D.; Grant, J.R.; Marcu, A.; Sajed, T.; Pon, A.; Liang, Y.; Wishart, D.S. PHASTER: A Better, Faster Version of the PHAST Phage Search Tool. Nucleic Acids Res. 2016, 44, W16-W21. [CrossRef]

59. Zhou, Y.; Liang, Y.; Lynch, K.H.; Dennis, J.J.; Wishart, D.S. PHAST: A Fast Phage Search Tool. Nucleic Acids Res. 2011, 39, W347-W352. [CrossRef]

60. Guy, L.; Kultima, J.R.; Andersson, S.G.E. GenoPlotR: Comparative Gene and Genome Visualization in R. Bioinformatics 2010, 26, 2334-2335. [CrossRef] [PubMed]

61. Abby, S.S.; Néron, B.; Ménager, H.; Touchon, M.; Rocha, E.P.C. MacSyFinder: A Program to Mine Genomes for Molecular Systems with an Application to CRISPR-Cas Systems. PLoS ONE 2014, 9, e110726. [CrossRef]

62. Daleke, M.H.; Ummels, R.; Bawono, P.; Heringa, J.; Vandenbroucke-Grauls, C.M.J.E.; Luirink, J.; Bitter, W. General Secretion Signal for the Mycobacterial Type VII Secretion Pathway. Proc. Natl. Acad. Sci. USA 2012, 109, 11342-11347. [CrossRef] [PubMed]

63. Kelley, L.A.; Mezulis, S.; Yates, C.M.; Wass, M.N.; Sternberg, M.J.E. The Phyre2 Web Portal for Protein Modeling, Prediction and Analysis. Nat. Protoc. 2015, 10, 845-858. [CrossRef] [PubMed]

64. McLaughlin, B.; Chon, J.S.; MacGurn, J.A.; Carlsson, F.; Cheng, T.L.; Cox, J.S.; Brown, E.J. A Mycobacterium ESX-1-Secreted Virulence Factor with Unique Requirements for Export. PLoS Pathog. 2007, 3, e105. [CrossRef]

65. Makarova, K.S.; Wolf, Y.I.; Alkhnbashi, O.S.; Costa, F.; Shah, S.A.; Saunders, S.J.; Barrangou, R.; Brouns, S.J.J.; Charpentier, E.; Haft, D.H.; et al. An Updated Evolutionary Classification of CRISPR-Cas Systems. Nat. Rev. Microbiol. 2015, 13, 722. [CrossRef]

66. da Silva Telles, M.A.; Chimara, E.; Ferrazoli, L.; Riley, L.W. Mycobacterium Kansasii: Antibiotic Susceptibility and PCR-Restriction Analysis of Clinical Isolates. J. Med. Microbiol. 2005, 54, 975-979. [CrossRef] [PubMed]

67. Santin, M.; Alcaide, F.; Benitez, M.A.; Salazar, A.; Ardanuy, C.; Podzamczer, D.; Rufi, G.; Dorca, J.; Martin, R.; Gudiol, F. Incidence and Molecular Typing of Mycobacterium Kansasii in a Defined Geographical Area in Catalonia, Spain. Epidemiol. Infect. 2004, 132, 425-432. [CrossRef] [PubMed]

68. Zhang, Y.; Mann, L.B.; Wilson, R.W.; Brown-Elliott, B.A.; Vincent, V.; Iinuma, Y.; Wallace, R.J. Molecular Analysis of Mycobacterium Kansasii Isolates from the United States. J. Clin. Microbiol. 2004, 42, 119-125. [CrossRef]

69. Bakuła, Z.; Safianowska, A.; Nowacka-Mazurek, M.; Bielecki, J.; Jagielski, T. Short Communication: Subtyping of Mycobacterium Kansasii by PCR-Restriction Enzyme Analysis of the Hsp65 Gene. BioMed Res. Int. 2013, 2013, 178725. [CrossRef]

70. Chen, J.M.; Zhang, M.; Rybniker, J.; Basterra, L.; Dhar, N.; Tischler, A.D.; Pojer, F.; Cole, S.T. Phenotypic Profiling of Mycobacterium Tuberculosis EspA Point Mutants Reveals That Blockage of ESAT-6 and CFP-10 Secretion In Vitro Does Not Always Correlate with Attenuation of Virulence. J. Bacteriol. 2013, 195, 5421-5430. [CrossRef]

71. Chen, J.M.; Boy-Röttger, S.; Dhar, N.; Sweeney, N.; Buxton, R.S.; Pojer, F.; Rosenkrands, I.; Cole, S.T. EspD Is Critical for the Virulence-Mediating ESX-1 Secretion System in Mycobacterium Tuberculosis. J. Bacteriol. 2012, 194, 884-893. [CrossRef]

72. Ates, L.S.; Brosch, R. Discovery of the Type VII ESX-1 Secretion Needle? Mol. Microbiol. 2017, 103, 7-12. [CrossRef] [PubMed]

73. Broset, E.; Martín, C.; Gonzalo-Asensio, J. Evolutionary Landscape of the Mycobacterium Tuberculosis Complex from the Viewpoint of PhoPR: Implications for Virulence Regulation and Application to Vaccine Development. mBio 2015, 6, e01289-15. [CrossRef]

74. Rohde, K.H.; Abramovitch, R.B.; Russell, D.G. Mycobacterium Tuberculosis Invasion of Macrophages: Linking Bacterial Gene Expression to Environmental Cues. Cell Host Microbe 2007, 2, 352-364. [CrossRef] [PubMed]

75. Homolka, S.; Niemann, S.; Russell, D.G.; Rohde, K.H. Functional Genetic Diversity among Mycobacterium Tuberculosis Complex Clinical Isolates: Delineation of Conserved Core and Lineage-Specific Transcriptomes during Intracellular Survival. PLoS Pathog. 2010, 6, e1000988. [CrossRef] [PubMed]

76. Ma, Z.; Strickland, K.T.; Cherne, M.D.; Sehanobish, E.; Rohde, K.H.; Self, W.T.; Davidson, V.L. The Rv2633c Protein of Mycobacterium Tuberculosis Is a Non-Heme Di-Iron Catalase with a Possible Role in Defenses against Oxidative Stress. J. Biol. Chem. 2018, 293, 1590-1595. [CrossRef]

77. Huynen, M.; Snel, B.; Lathe, W.; Bork, P. Predicting Protein Function by Genomic Context: Quantitative Evaluation and Qualitative Inferences. Genome Res. 2000, 10, 1204-1210. [CrossRef]

78. Sievers, F.; Wilm, A.; Dineen, D.; Gibson, T.J.; Karplus, K.; Li, W.; Lopez, R.; McWilliam, H.; Remmert, M.; Söding, J.; et al. Fast, Scalable Generation of High-quality Protein Multiple Sequence Alignments Using Clustal Omega. Mol. Syst. Biol. 2011, 7. [CrossRef]

79. Kurtz, S.; Phillippy, A.; Delcher, A.L.; Smoot, M.; Shumway, M.; Antonescu, C.; Salzberg, S.L. Versatile and Open Software for Comparing Large Genomes. Genome Biol. 2004, 5, R12. [CrossRef]

80. Delcher, A.L.; Phillippy, A.; Carlton, J.; Salzberg, S.L. Fast Algorithms for Large-Scale Genome Alignment and Comparison. Nucleic Acids Res. 2002, 30, 2478-2483. [CrossRef] 